\title{
ALGUNOS FRAGMENTOS DE MÚSICA ESPAÑOLA POR LA PIANISTA PILAR BAYONA. ANALISIS ESPECTROGRÁFICO DE SU INTERPRETACIÓN
}

\author{
SOME FRAGMENTS OF SPANISH MUSIC PLAYED BY THE PIANIST PILAR \\ BAYONA. SPECTROGRAPHIC ANALYSIS OF ITS INTERPRETATION
}

\author{
Rubén Lorenzo Gracia \\ Universidad Politécnica de Valencia \\ rubenlorenzopiano@gmail.com \\ ORCID iD: https://orcid.org/0000-0003-4884-5427
}

\begin{abstract}
Resumen
Pilar Bayona fue una figura relevante del piano en España durante el siglo XX que no ha sido estudiada hasta la fecha, desde el punto de vista técnico e interpretativo. Especialmente interesantes fueron sus interpretaciones de la música española.

En el presente trabajo realizo un análisis de algunos fragmentos que la pianista ha dejado grabados de este estilo. En mi análisis utilizo una metodología propia que permite realizar un diagnóstico objetivo de su interpretación mediante espectrogramas sonoros, imágenes y otras gráficas derivadas de ellos.
\end{abstract}

\section{Palabras clave}

Espectrograma, interpretación musical, interpretación pianística, música española

\section{PILAR BAYONA Y LÓPEZ DE ANSÓ (*ZARAGOZA, 16.09.1897; †IBÍD., 13.12.1979). BREVE BIOGRAFÍA.}

Buena parte de los datos y de las anécdotas vitales de la pianista, pueden encontrarse en la actualidad en otros trabajos disponibles que, por otra parte, se han centrado mucho más y con mayor detalle en cuestiones personales y sociales. A ellos remito al lector para ampliar o pormenorizar cuantos detalles estime convenientes ${ }^{1}$.

1 A este respecto, la obra más completa sobre la vida de la pianista hasta la fecha está realizada por los responsables del Archivo Pilar Bayona.

\begin{abstract}
Pilar Bayona, one of the foremost Spanish pianists of the twentieth century, has been the baseline of this study as her figure has hitherto not been studied from the technical and interpretive perspective. Their interpretations of Spanish music were particularly interesting.

The aim of this work is to analyse some of recorded fragments from this musical style she has left. To undertake this task I use my own work methodology that allows to make objective assessments of her individual performance using sound spectrograms, pictures and other graphs derived from them.
\end{abstract}

\section{Key words}

Spectrogram, musical performance, piano performance, experimental teaching, Spanish music

Me limitaré a trazar unas breves líneas principales de su biografía, centrándome fundamentalmente en aspectos profesionales y artísticos de relevancia en la vida de la pianista que faciliten una mejor comprensión de su aportación al ámbito de la interpretación musical pianística en España.

No obstante, existen diversos libros, articulos y trabajos anteriores que han aportado bastante información sobre la pianista. Véase BAYONA Y GÓMEZ (2015). BROTO SALAMERO (1986). PÉREZ-LIZANO FORNS (1991). BAYONA DE LA LLANA ( 2004). VV. AA. (2009). SOPEÑA IBÁÑEZ (1982). 
Pilar Bayona desarrolló su actividad musical fundamentalmente en dos etapas diferenciadas, marcadas como eje principal en la Guerra Civil española. Una primera etapa, antes de la guerra, en la que podrían distinguirse a grandes trazos tres períodos: $a$ ) hasta 1920 (con su etapa de infancia y formación musical, los años de 1914 a 1920); b) la década de 1920; (inicio de su carrera profesional y proyección nacional e internacional) y c) la década de 1930 (con su presencia en la Residencia de Estudiantes de Madrid, y la propia contienda). Mientras que, después de la guerra, podrían diferenciarse tres etapas: $d$ ) la posguerra (hasta 1950, Radio Zaragoza); e) la década de 1950 (una década de gran actividad) y $f$ ) desde 1960 (como reconocimiento a su labor); hasta sus últimos años y su muerte en 1979.

Sin antecedentes musicales en la familia, su padre, Julio, era profesor de matemáticas y aficionado a la música, mientras que su madre, Sara, tocaba el piano del que disponía la familia. Pronto comenzó a estudiar con dos hermanos catalanes - que habían estudiado con el célebre pianista Joaquín Malats $(* 1872 ; \dagger 1912) —$, José y Ángeles Sirvent, que entonces residían en Zaragoza. Niña prodigio, hizo unas cuantas tempranas actuaciones públicas (ya en 1905, en el Teatro Principal de la capital aragonesa y en el Gran Casino), si bien debutó como solista en concierto el 20.12.1907, en la Sociedad Filarmónica de Zaragoza, en un programa en el que actuó junto al cuarteto del violinista zaragozano Teodoro Ballo $(* 1866 ; \dagger 1962)$.

Se considera que inicia su carrera profesional en 1912, actuando en el primer concierto de la Asociación Bretoniana, dirigido por el propio maestro Tomás Bretón $(* 1850 ; \uparrow 1923)$, procediendo enseguida a dar recitales en Oviedo, Pamplona y Madrid, ofreciendo una audición en el Palacio Real para las infantas. Inicia asimismo por entonces su colaboración con José $\mathrm{M}^{\text {a }}$ Usandizaga $(* 1887$; $\dagger 1915)$ y ofrece conciertos en San Sebastián, bajo la dirección del eminente Enrique Fernández Arbós (*1863; †1939). A partir de entonces, inicia una actividad concertística que, siempre desde Zaragoza, le llevará por toda España (Barcelona, Alicante, Bilbao...), coincidiendo con compositores (algunos de los cuales le dedicaron sus composiciones), intérpretes y críticos destacados de la esfera nacional, como Eduardo López-Chávarri $(* 1871 ; \dagger 1970)$, Joaquín Turina $(* 1882 ; \dagger 1949)$, Jesús Guridi $(* 1886 ; \dagger 1961)$, Óscar Esplá (*1886; $\dagger 1976)$, Arthur Rubinstein $(* 1887 ; \dagger 1982)$, Adolfo Salazar $(* 1890 ; \dagger 1958)$, Rafael Martínez $(* 1895 ; \dagger 1953)$, etc. Todo esto, unido a su juventud, le sirvió para darse a conocer, granjeándose cierto reconocimiento público. Apareció por entonces reflejada, con relativa asiduidad, en entrevistas publicadas en revistas y artículos de prensa, y así por ejemplo en Lira Española, El Bretoniano, Correspondencia,
La Linterna, Juventud, Aragoneses contemporáneos, Lira Española, Pluma Aragonesa, Mundo Gráfico. También en esos años establece su amistad con el cineasta Luis Buñuel $(* 1900 ; \uparrow 1983)$-alumno de su padre, Julio Bayona-, y con el historiador y literato José Camón Aznar $(* 1898 ; † 1979)$. Enrique Jardiel Poncela $(* 1901 ; \dagger 1952)$ glosó sobre ella algunos poemas en 1915 .

En términos generales, puede decirse que en este periodo su labor fue (más allá de sus estudios con Ángeles Sirvent), básicamente autodidacta y autónoma, como revela el hecho de que ejerciera como su propia representante para gestionar sus actividades profesionales. En la década de 1910 incorpora a su repertorio, algunas primeras audiciones, que incluyen autores entonces recientes y aun coetáneos como Claude Debussy, Enrique Granados, Manuel de Falla u Óscar Esplá, en un compromiso con la difusión de la música contemporánea, especialmente española, que le iba a caracterizar a lo largo de toda su trayectoria profesional.

En la década de los años 20, como pianista provista de un criterio propio, especialmente intuitiva, prosigue con sus conciertos a nivel nacional, ofreciendo primeras audiciones de compositores foráneos (Mili Balákirev o Maurice Ravel) y de autores españoles (como Isaac Albéniz, Manuel de Falla, Ernesto Halffter, el padre Donostia o Federico Mompou). En 1924 viaja a Alemania, actuando en Stuttgart y Berlín, donde dio a conocer la música contemporánea española (Albéniz, Granados, Falla, Ernesto Halffter, Usandizaga, Donostia, Esplá, Mompou...). En 1925, la Asociación de Profesores Músicos y la Agrupación Artística Aragonesa, ambas de Zaragoza, le nombran "Socia de Honor", idéntico honor al recibido, al año siguiente, de la Orquesta Sinfónica de Zaragoza. En 1927, también participó en algunas sesiones camerísticas zaragozanas, con un par de conciertos junto a su amigo, el citado violinista Rafael Martínez, dando a conocer algunas composiciones en primera audición e incluyendo en su repertorio obras del darocense Ángel Mingote Lorente $(* 1891 ; \dagger 1961)$. En 1928 estrena en la capital del Turia el Concierto Valenciano de Eduardo López-Chávarri $(* 1871 ; \dagger 1970)$, y ese mismo año tiene ocasión de conversar brevemente en la capital aragonesa con Maurice Ravel $(* 1875 ; \dagger 1937)$,-entonces de gira por España para presentar sus obras-, tras un concierto con un tipo de música, entonces, todavía poco apreciado por la audiencia local.

Por esos años se destaca como famosa en los círculos intelectuales de la ciudad; le dedican su atención tanto poetas -v.g., Pablo Cistué de Castro, barón de La Menglana (*1901; †1980)-, como diferentes artistas plásticos, y así por ejemplo, le regalaron obras o le retrataron el modernista José Zamora $(* 1889 ; \dagger 1971$ ?), el químico e ilustrador gráfico Luis Pablo Sanz Lafita $(* 1902 ; \dagger 1996)$-quien, 
compañero de su hermano Julio, le dedicó algunas divertidas caricaturas-, o Guillermo Pérez Baylo $(* 1911 ; \nmid 2000)$. También el músico navarro Fernando Remacha $(* 1898$; $\dagger 1984)$ le dedica por entonces algunos ejemplares de sus composiciones.

Aunque el estallido de la Guerra Civil marcará un antes y un después en el devenir histórico y social del país, y aun abrirá un paréntesis en su actividad profesional pública, en la década de 1930 Pilar Bayona siguió ofreciendo conciertos en varias ciudades del país, tanto en recital como con orquesta, actuando bajo la dirección de maestros tales como Luis Aula $(* 1876 ; \dagger 1945)$, el padre José María Nemesio Otaño y Eguino $(* 1880 ; \nmid 1956)$, Jesús Arámbarri y Gárate $(* 1902 ; \dagger 1960)$, o César Mendoza Lasalle $(* 1910 ; \dagger 1999)^{2}$.

En Madrid, el 13.03.1931, ofrece un breve recital con motivo de la toma de posesión del general Manuel Aznar como presidente de la Asociación de Cultura Musical, apareciendo en portada del diario $A B C$, y ofrece un concierto en la Sociedad Filarmónica de Madrid, junto al cuarteto de Rafael Martínez, con el que estrena el Cuarteto con piano $n .^{\circ} 2$, Op. 67, de Joaquín Turina $(* 1882 ; \dagger 1949)$. Ese mismo año, comparte programa en Zaragoza en un conciertofestival benéfico junto al tenor Miguel Fleta $(* 1897 ; \uparrow 1938)$. Y se ha localizado un ejemplar de 1931 de la revista Tesoro Sacro Musical, en la que se editan dos partes de la Misa para teclado del ya citado compositor Ángel Mingote, dedicados a la pianista.

$\mathrm{Al}$ año siguiente, la Sociedad de Conciertos de Zaragoza le nombra "Socia de Honor". Por entonces también, el novelista zaragozano Tomás Seral y Casas $(* 1908 ; \nmid 1975)$ le dedica una de las poesías de su libro Poemas del amor violento y le menciona en varias notas de Noreste, revista literaria en la que colaboró con el escritor Ildefonso Manuel Gil $(* 1912 ; \nmid 2003)$.

En 1936, reside temporalmente en Madrid, frecuentando la Residencia de Estudiantes, donde prepara sus conciertos, en unas veladas musicales a las que asisten, entre otros, el compositor chileno Acario Cotapos Baeza (*1889;

2 Célebre por haber dirigido el estreno -con Regino Sainz de la Maza como guitarra solista-, en el Palau de la Música de Barcelona, en 1940, del Concierto de Aranjuez del maestro Joaquín Rodrigo. [Impulsada su carrera inicialmente gracias a su tío, el director de renombre José Lassalle, se trasladó en 1942 a Latinoamérica, recorriendo durante las dos décadas siguientes todo aquel continente dirigiendo orquestas de prestigio. Dirigió también abundantes ballets, y tuvo relación con prestigiosos artistas, para finalizar su carrera en España en la década de 1960, dirigiendo numerosas zarzuelas, tras de lo cual se retiró a la localidad francesa de Gros Rouvre, en Montfort L'Amaury]. $\dagger 1969)$, el crítico musical Adolfo Salazar $(* 1890 ; \dagger 1958)^{3}$, Federico García Lorca $(* 1898 ; \dagger 1936)^{4}$, los hermanos Luis -el célebre director de cine- $(* 1900 ; \uparrow 1983)$ y Alfonso Buñuel Portolés $(* 1915 ; \dagger 1961)$-arquitecto y artista del surrealismo-, el escritor e intelectual José ["Pepín”] Bello Lasierra (*1904; †2008), o el arquitecto Juan Pérez Páramo.

En mayo, participa en una cena en homenaje al pintor Hernando Viñes Soto $(* 1904$; $† 1993)$-junto a los anteriormente citados y a otros artistas-, que aparecen retratados en una famosa fotografía del entonces grupo intelectual activo en torno a la Residencia de Estudiantes madrileña ${ }^{5}$.

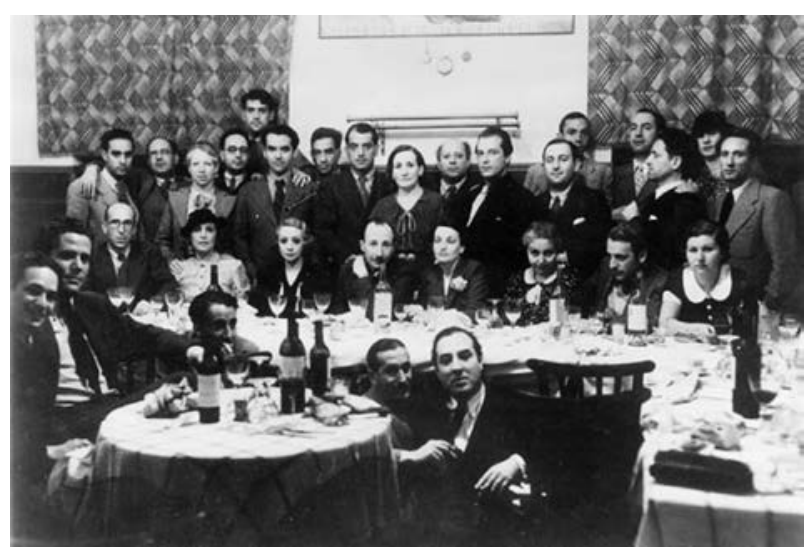

Fig. 1. Homenaje al pintor Hernando Viñes, con Adolfo Salazar, Federico García Lorca, Luis Buñuel, Rafael Alberti, Miguel Hernández, Pablo Neruda... (Madrid, mayo de 1936). [Archivo de la Residencia Estudiantes, CSIC].

En la década de 1930 le dedican ejemplares de sus partituras compositores como el ya ampliamente citado Óscar Esplá -alicantino-, así como el valenciano Manuel Palau Boix $(* 1893$; $† 1967)$, el chelista y compositor catalán Gaspar Cassadó $(* 1897 ; \dagger 1966)$, el madrileño Rodolfo Halffter

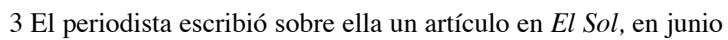
de 1936, en el que analiza la personalidad musical y temperamento de la pianista.

4 El célebre escritor granadino le regaló un ejemplar de la primera edición de Llanto por Ignacio Sánchez Mejías, con dedicatoria autógrafa y una peculiar firma, artísticamente rubricada. Pilar Bayona poseyó asimismo otro autógrafo original de García Lorca: un ejemplar de Mariana Pineda (de 1928), dedicado a Lorenzo Dueñas, que probablemente le regaló éste -siendo periodista de Granada-, que caricaturizó por otra parte a la pianista en 1931 y le regaló diversos dibujos.

5 Y así por ejemplo, aparecen en la foto, junto a los citados y a la pianista zaragozana, Rafael Alberti $(* 1902 ; \dagger 1999)$, Pablo Neruda $(* 1904 ; \dagger 1973)$, o Miguel Hernández $(* 1910 ; \dagger 1942)$, entre otros varios. 
(*1900; †1987), o los valencianos Vicente Asencio y Ruano $(* 1908 ; \dagger 1979)$, y Matilde Salvador Segarra $(* 1918 ; \dagger 2007)$. También el entonces maestro de capilla de El Pilar, Gregorio Arciniega Mendi $(* 1886 ; \dagger 1967)$, le dedica el manuscrito original de su transcripción de las obras de Gaspar Sanz para piano. Y el arquitecto Manuel Martínez Chumillas (*1902; $\dagger 1986$ ) le dedica su primera composición musical.

En plena contienda se funda Radio Zaragoza (1938), participando Pilar Bayona en su concierto de inauguración, junto a Luis Aula como director. A partir de entonces, Pilar Bayona iba a colaborar asiduamente con dicha emisora ofreciendo conciertos en directo, en unas interpretaciones que se convirtieron en tradicionales en las ondas de la capital aragonesa, en las cuales se desplegó un amplísimo repertorio. Así, en los años 1938-1943 Pilar Bayona interpretó semanalmente música de cámara y desde 1943 hasta su muerte en 1979, intervino en la radiodifusión de obras como solista.

Unos cuantos hechos importantes de carácter personal sucedieron en la vida de Pilar Bayona en torno a la década de 1940: por un lado, el fallecimiento de su padre en 1938, y el de su madre, tres años después, en 1941; y por otro lado, se le diagnosticó una enfermedad de la columna, que iba a provocar que su talla física fuera encogiendo progresivamente a lo largo de los años.

En sus actuaciones en la recién creada Radio $\mathrm{Za}$ ragoza, acuden a escucharla el grupo intelectual de aquellos años: Alfonso Buñuel, el catedrático universitario Federico Torralba Soriano $(* 1913 ; \dagger 2012)^{6}$, el poeta Juan Eduardo Cirlot Laporta $(* 1916 ; \uparrow 1973)^{7}$, el historiador del arte Julián Gállego Serrano (*1919; †2006), el polifacético lingüista Luis García-Abrines Calvo $(* 1923)^{8}$, el arquitecto Juan Pérez Páramo, el crítico musical Eduardo Fauquié $(\dagger 1985)^{9}$ o el ingeniero José $\mathrm{M}^{\mathrm{a}}$ García Gil ${ }^{10}$.

Aparte de la densa tarea iniciada en Radio Zaragoza, en esta década emprendió otra actividad, de carácter pedagógico, que le acompañó ya hasta el final de su vida. Hacia 1944, gracias a su amistad con Federico Sopeña Ibáñez

6 Decano de los historiadores y críticos de arte españoles, era un destacado estudioso del arte oriental, así como experto en la obra de Francisco de Goya y en arte contemporáneo.

7 También crítico de arte, su interés por la música y la amistad con la pianista, le llevaron a dedicarle su libro de poemas Pájaros tristes (1942), inspirado en la interpretación de la artista zaragozana de la obra homónima de Maurice Ravel.

8 Artista, musicógrafo, e investigador de la lengua española en Estados Unidos.

9 Ejerció la crítica musical en el diario Heraldo de Aragón.

10 Marido de la célebre bailarina clásica María de Ávila, circunstancia que facilitó la gran amistad entre ésta y Pilar Bayona.
$(* 1917 ; \dagger 1991)^{11}$, comenzó a tomar parte en un ciclo de conferencias-concierto (incluido en la Semana Musical de los cursos de verano de la Universidad de Zaragoza en Jaca), participación periódica que se iba a prolongar a lo largo de 34 años.

Estos cursos ofrecían un tipo de conferencias que se dedicaban a diversas épocas y autores, tratando de cubrir el amplio espectro cronológico de la música española, además de mostrar la producción de diferentes compositores extranjeros.

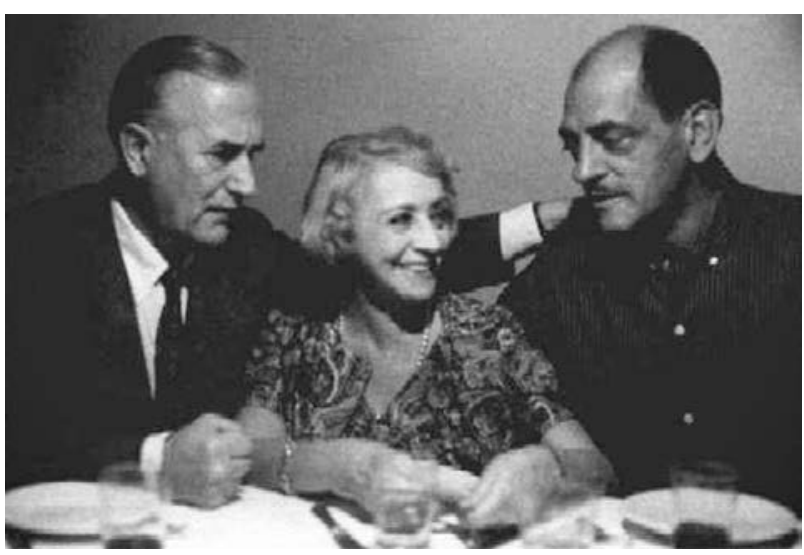

Fig. 2. Pepín Bello, Pilar Bayona y Luis Buñuel.

En 1946 y 1947, Pilar Bayona prosiguió relacionándose con el mundo de las artes plásticas y las letras. Aparecieron en esa época sus contactos más o menos frecuentes con el pintor José Mompou Dencausse $(* 1888 ; \dagger 1968)$-hermano del compositor Federico-, con el pintor y arquitecto Santiago Lagunas $(* 1912 ; \dagger 1995)^{12}$, y con el poeta, periodista y dibujante

11 Filósofo, crítico musical, musicólogo y docente, además de gestor cultural, Historiador del arte y museógrafo, licenciado en Derecho y doctor en Teología por la Universidad Gregoriana. De vocación sacerdotal tardía, fue nombrado por Pablo VI prelado doméstico del papa con título de monseñor. Miembro de la Real Academia Española (1958). Llegó a ser director de la Academia Española de Bellas Artes en Roma (1977-1981), del Museo del Prado (1981-1983), y de la Real Academia de Bellas Artes de San Fernando (1988-1991). En su faceta estrictamente musical fue Secretario de la Comisaría Nacional de la Música con Joaquín Turina (1940-1943), crítico musical de los diarios Arriba y $A B C$, catedrático de Estética e Historia de la Música, y director de la revista Música del conservatorio madrileño, además delegado del Gobierno en los conservatorios españoles y director del Conservatorio de Madrid (1951-1956). Fue investigador del Instituto Español de Musicología del CSIC (responsable de su sección de música contemporánea, en Madrid), y Comisario General de la Música (1971-1972). Entre otros trabajos, publicó sobre Manuel de Falla, Stravinsky y Turina, y contribuyó de forma pionera a difundir en España la música de Gustav Mahler. VV.AA. (2000).

12 Se conserva una felicitación navideña a Pilar Bayona de este pintor. 
Gil Comín Gargallo (*1899; $\dagger 1976)^{13}$-sobrino del célebre escultor Pablo Gargallo $(* 1881 ; \dagger 1934)-$.

El Ayuntamiento de Zaragoza le concede su reconocimiento como "Hija Predilecta" de la ciudad en $1947^{14}$, y recibe también el nombramiento de "Socia de Honor" de la Sociedad Filarmónica zaragozana, en un acto celebrado en su homenaje.

Su principal actividad de conciertos en esta década, la realiza en España, Francia, Portugal, y con bastante frecuencia, en Marruecos, colaborando además con el ya citado director de orquesta Jesús Arámbarri y Gárate, así como con los catalanes Eduardo Toldrá Soler $(* 1895 ; \uparrow 1962)$ y Carlos Suriñach Wrokona $(* 1915 ; \dagger 1997)$, y con el belga Steven Candae ${ }^{15}$.

La década de 1950 representa la cumbre de su carrera artística y el inicio del reconocimiento a su labor. Tres actividades, fundamentalmente, marcan el desarrollo de esta etapa en la vida de la pianista. Por un lado, la intensa actividad de conciertos ${ }^{16}$, por otro lado, la aparición de sus primeras grabaciones discográficas comerciales, y finalmente, el desarrollo de su faceta como docente.

En lo que respecta a sus actuaciones en concierto en Zaragoza, coincidiendo con los años de mayor auge de la Sociedad Filarmónica, un grupo de melómanos encabezados por los ya citados Luis García Abrines y Eduardo Fauquié, además de por Fausto Gavín Bueno (†1966) y Manuel Derqui Martos (*1921; †1973), crearon entonces una sección de “música de cámara y moderna" que se denominó Sansueña ${ }^{17}$. Pilar Bayona actuaría allí de manera continuada, ofreciendo recitales de carácter monográfico, de autores como Isaac Albéniz, Maurice Ravel, Manuel de Falla u Óscar Esplá.

13 Le dedicó un poema que se publicó en prensa.

14 No obstante, la entrega del título de "Hija Predilecta" de Zaragoza no se realizó hasta octubre de 1955 , en un concierto de gala celebrado en el Palacio de la Aljafería de la capital aragonesa.

15 Fundador de la Orquesta Filarmónica Real de Flandes (De Filharmonie / Koninklijke Filharmonie van Vlaanderen / Royal Flemish Philharmonic), en primer término bajo el nombre de Antwerpse Philharmonie (o Filarmónica de Amberes).

16 Más allá de la actividad continuada de actuaciones realizada desde los comienzos de Radio Zaragoza, sólo hasta 1957 realizó 150 conciertos.

17 Nombre mitológico de Zaragoza en el romancero. Según el historiador Ramón Menéndez Pidal, el nombre de Sansueña procedería del francés "Sansoigne" (= "Sajonia"). En El retablo de Maese Pedro de Manuel de Falla, cuyo libreto se inspira en un episodio del Quijote, Cervantes identifica Sansueña con la ciudad de Zaragoza, según la opinión de la época. El concierto de inauguración de esta "sección de música de cámara de la Sociedad Filarmónica", corrió a cargo de Pilar Bayona, que precisamente lo dedicó monográficamente a este compositor. Sansueña sólo permanecería activa desde 1950 hasta 1954, organizando un total de 19 conciertos. Véase, MENÉNDEZ PIDAL (1953): 256. PÉREZ-LIZANO (1991): 38.
Pero la piansta repitió estos recitales de carácter monográfico en varias ciudades de la geografía española, realizando, una vez más, algunas primeras audiciones de autores españoles. En esta época recibió partituras dedicadas de Óscar Esplá, Manuel Palau Boix $(* 1893 ; \dagger 1967)$, José Moreno Gans $(* 1897 ; \dagger 1976)$, Salvador Bacarisse Chinoria (*1898; $\dagger 1963)$, Joaquín Rodrigo Vidre $(* 1901 ; \dagger 1999)^{18}$, Elena Romero $(* 1907$; $\dagger 1996)$, Luis de Pablo Costales $(* 1930)$ y Jaime Nogales Bello ${ }^{19}$. De estas obras daría primeras audiciones -estrenando muchas de ellas-, como en el caso ejemplo de la Sonata Española, Op.53 "Homenaje a Chopin" (que tocó en 1951$)^{20}$, o de la Sonata del Sur para piano y orquesta, Op.52 (que interpretó en 1954, junto a la Orquesta Municipal de Barcelona), ambas obras de Óscar Espláa ${ }^{21}$; o incluso en el estreno que realizó en Alicante, en 1956, de la Fantasía homenaje a Walt Disney, para piano y orquesta, del compositor vasco Jesús Guridi.

Muy destacadas fueron también las interpretaciones que abordó públicamente durante estos años de la suite $I b e$ ria de Isaac Albéniz, que ofreció en su versión completa, con todos los números, en cuatro ocasiones ${ }^{22}$. De hecho, y como consecuencia de su fama como intérprete de música española y su destacada labor como divulgadora de la misma, el pianista francés de origen búlgaro Alexis Weissenberg (*1929; $\dagger 2012$ ), la propuso a la casa discográfica francesa Lumen, para que fuera ella quien realizara la grabación de la suite Iberia de Albéniz, que, efectivamente, registró en $1955^{23}$. En esta misma línea, en 1957 y 1958, grabó para el sello Hispavox dos discos dedicados a la música de Óscar Esplá y de Jesús Guridi.

Pero estos años fueron importantes también en lo que respecta a su dedicación a la enseñanza. En esta década, coBayona.

18 Que dedicó su quinta Sonata de Castilla, en La Mayor, a Pilar

19 Que, además de compositor, ejerció como crítico musical de la revista Ritmo. Dedicó a Pilar Bayona su obra El amor y la campesina.

20 Según Manuel Derqui, la Sonata española de Oscar Esplá fue una obra compuesta expresamente para ella. En 1952 también estrenaría otra obra de Esplá: La Lírica Española I. Sobre la Sonata Española, fue compuesta en 1949, por encargo de la UNESCO, siendo estrenada por Arnaldo Estrella en la Sala Gaveau de París, el 03.10.1949. No obstante, Pilar Bayona llegaría a tocar en público esta obra en más de treinta ocasiones, especialmente en el año 1951, siendo su principal difusora. Véase: DERQUI (1982): 120-121. IGLESIAS (1962). IGLESIAS (1994).

21 Compuesta en el año 1943, y con versión definitiva de 1945, esta obra, dedicada al pianista zaragozano Eduardo del Pueyo, fue estrenada por este último el 11.10.1945.

22 Desde 1912, Pilar Bayona ya había interpretado en numerosas ocasiones piezas sueltas de esta suite. Como también iba a ofrecer integrales de la Iberia en la década de 1960.

23 Aunque no llegaron a editarse más que siete de las doce piezas. 
menzó a dar las primeras clases de piano de su vida, en concreto, a sus sobrinos, finalizando con sus primeras clases en el Conservatorio "Pablo Sarasate" de Pamplona. Con motivo de la inauguración de dicho centro, y cuando Fernando Remacha se hizo cargo de la dirección del nuevo centro, Pilar intervino en cinco conferencias-concierto ${ }^{24}$.

Entretanto, Pilar Bayona sigue en contacto y amistad con la intelectualidad aragonesa: es una gran aficionada a la lectura, al cine, a las exposiciones, y - lo que es más raro en un pianista - acude a todos los conciertos que puede, allí donde se encuentre ${ }^{25}$.

Pilar Bayona vivió con su hermana Carmen hasta el final de sus días, y el año 1960 comenzó con el traslado de su residencia habitual. Continuaba entonces con su actividad iniciada en la década anterior, ofreciendo conciertos por toda la península y Marruecos, además de proseguir con su labor en Radio Zaragoza, en los cursos de verano universitarios de Jaca, y con sus clases en el Conservatorio de Pamplona. Así por ejemplo, en enero de 1961 estrena en Málaga la Rapsodia de Estella de Fernando Remacha (compuesta en 1958), bajo la dirección de Rafael Frübeck de Burgos $(* 1933 ; \nmid 2014)$, de la que realizaría posteriormente la primera audición en Madrid.

Desde entonces y hasta el final de sus días, iba a recibir numerosos homenajes, así como el reconocimiento generalizado, desde distintos estamentos, a la labor de toda su vida: en 1963, - por concurso de méritos, y sin titulación acadé-

24 Otros actos estuvieron a cargo del padre Samuel Rubio Calzón (*1912; †1986) conocido musicólogo y organista, y de Ricardo Urgoiti Somovilla (*1900; †1979), empresario de comunicación, con quien mantuvo gran amistad toda su vida. En 1958, y con ocasión de la creación de la Cátedra "Félix Huarte", repitió conciertos y cursos en Pamplona. Esta cátedra facilitó la organización de unos cursos anuales, a los que asistieron relevantes personalidades musicales, tales como el propio Fernando Remacha, Óscar Esplá, el compositor y pedagogo Goffredo Petrassi (*1904; †2003), el intérprete de música antigua, pianista y clavicembalista alemán Franzpeter Goebels $(* 1920 ; \dagger 1988)$, el crítico musical, Enrique Franco Manera $(* 1920 ; \dagger 2009)$, o el famoso guitarrista Narciso [García] Yepes $(* 1927$; $\uparrow 1997$ ). Como profesora del Conservatorio de Pamplona, Pilar Bayona impartiría clases hasta 1975. [Hablaré de ello más adelante].

25 A este respecto Federico Sopeña dice lo siguiente: “[...] Pilar pertenecía a la minoría - bastante exigua- de músicos que oyen a los otros, que va a los conciertos, que dicen su parecer sin ambages y rodeos. Insisto en que esa minoría es bastante exigua, limitada a quienes tienen dos cualidades no contrapuestas sino complementarias: seguridad en sí mismo y antenas abiertas para enriquecerse con lo que hacen los otros, cuando sean grandes. Por eso Pilar ha tenido tantos amigos, entre los músicos, especialmente entre los pianistas jóvenes... [...]. Tal vez sea por ello que Manuel Derqui le dedica uno de sus cuentos; Julián Gallego, un ejemplar de Fedra, obra que recibe el premio A. Balaguer de Teatro; Pilar Aranda le hace un retrato a la acuarela; su amigo García-Abrínes le regala uno de los cuadros de su exposición en la sala Reyno, en 1951, y le dedica, junto a Adolfo Salazar, la edición facsímil de Instrucción de música sobre la guitarra Española de Gaspar Sanz, que coordina en 1952". Véase: SOPEÑA IBÁÑEZ (1982): 10. mica alguna-, el Ministerio de Educación le nombra profesora de la especialidad de Virtuosismo en el Conservatorio de Zaragoza, puesto que iba a ejercer hasta su fallecimiento. $\mathrm{Al}$ año siguiente, 1964, el Ayuntamiento de Zaragoza pone su nombre a una calle de la ciudad. Y en 1968, la "Institución Fernando el Católico" de la Diputación de Zaragoza, le concede el Premio "San Jorge"26. Al poco, en mayo de 1969, ingresa en la Real Academia de Nobles y Bellas Artes de San Luis, de Zaragoza, reconocimiento al que se unirían otros homenajes, como los ofrecidos por la Asociación "Amigos de la Música", la Sociedad "Juventudes Musicales", o el Club "Calibo"27.

Por otra parte, los numerosos galardones y nombramientos que recibe continúan en la década de 1970: un homenaje conjunto al lado de su amiga la maestra de danza y bailarina clásica María de Ávila $(* 1920 ; \uparrow 2014)$, en 1972 ; el Premio "Conservatorio de Zaragoza", en 1973; el homenaje de la Agrupación Provincial de Radio y Televisión, en 1973; nombrada personalidad "Popular" por el periódico El Noticiero, en 1974; "Hija Adoptiva" de su pueblo natal, Cosuenda, en 1975, donde se puso su nombre a la calle en la que se encontraba la casa materna de la pianista; medalla de Radio Zaragoza con motivo del $40^{\circ}$ aniversario de la emisora en 1977; y "Consejera de Número" de la Institución "Fernando el Católico" en 1978.

En la década de 1960 graba asiduamente para Televisión Española, actuando para distintos programas musicales en $1960,1964,1965$ y $1968^{28}$.

En el año 1968, actúa en la Academia de Bellas Artes de San Fernando de Madrid, interviniendo en una

26 Con acto de entrega glosado por el catedrático universitario Federico Torralba Soriano.

27 La asociación de Juventudes Musicales, con origen en Francia y Bélgica (fundadas en 1940 por Marcel Cuvelier y René Nicol, respectivamente), es un grupo creado con el fin de fomentar la afición por la música y apoyar a los intérpretes noveles. La sección española se había creado en 1956. En Zaragoza, fueron sus fundadores, en 1957, Asunción Baselga e Ignacio Seral, entre otros. En la capital aragonesa, antes de 1993 (fecha en que se inauguró el Auditorio de la ciudad), los foros que ofrecían conciertos de música se correspondían más bien con entidades privadas, como la citada Juventudes Musicales o la Sociedad Filarmónica. Muchos de estos conciertos tenían lugar en lugares reducidos (colegios mayores...), e incluso en pisos, como por ejemplo, en Calibo (calle Blancas, 2), que fue un club cultural zaragozano de carácter privado, que ofrecía distintas actividades, tales como conferencias y conciertos. También tenían una actividad frecuente el Círculo $\mathrm{Me}$ dina, situado en la calle del Coso, número 86, o el Museo "Camón Aznar", de Ibercaja (calle Espoz y Mina, 23), así como la sala de la CAI (Caja de Ahorros de la Inmaculada, Paseo de la Independencia, 2). Véase la entrada correspondiente a "Juventudes musicales", de la GEA (Gran Enciclopedía Aragonesa, on-line) [actualizada en 2006].

28 A día de hoy no se ha recuperado ninguna de las grabaciones que realizó para la televisión. 
conferencia-concierto junto al padre Federico Sopeña, con ocasión del centenario de Enrique Granados. Y también estrena ese mismo año, en Barbastro (Huesca), la obra Carrillon, de Joaquín Broto Salamero $(* 1921 ; \dagger 2006)^{29}$, mientras los compositores Javier Alfonso $(* 1904 ; \dagger 1988)^{30}$ y Antón Roch $(* 1916 ; \dagger 1987)^{31}$ le dedican ejemplares de sus obras.

En Madrid actuaría varias veces más durante esta década, y así, ofrece varios recitales monográficos en el Museo Romántico de Madrid, sobre autores como Robert Schumann, Claude Debussy o Isaac Albéniz. También en la capital de España ofrece en el Ministerio de Turismo, en 1968, un concierto que, grabado en directo por Radio Nacional, sería editado en disco de vinilo, y posteriormente galardonado con el Premio "Ministerio de Cultura" en 1981.

Pero sus viajes no cesaban, pues participaría también durante dos años en los cursos de verano de la Universidad "Menéndez Pelayo" de Santander, período del que se conserva un retrato suyo a cargo del pintor Benjamín Palencia $(* 1894 ; \dagger 1980)$.

Su intensa actividad como pianista continuó en la década de 1970 hasta el final de su vida. Actuó en más de veinte ciudades españolas, y en Zaragoza participó varias veces en unas populares Jornadas Culturales del Ayuntamiento, actuando en diversas ocasiones con orquesta, dirigida por Vicente Spiteri Galiano $(* 1917 ; \nmid 2003)^{32}$. En esta misma

29 Joaquín Broto, discípulo de Cristóbal Taltabull, fue organista titular de la catedral metropolitana de La Seo de Zaragoza, profesor del conservatorio de música de la misma ciudad (desde 1963) y compositor. Anteriormente, había sido premio extraordinario en Órgano del Conservatorio de Madrid, organista de la catedral de Barbastro, y maestro de capilla de las catedrales de Barcelona y Santiago de Compostela. Fue académico de la de Nobles y Bellas Artes de San Luis de Zaragoza desde 1969.

30 Pianista y compositor madrileño (Premio Nacional de Piano, 1940; Premio de Musicología, 1946; y Premio "Eduardo Aunós" para pianistas, 1947), discípulo de José Tragó, Bartolomé Pérez Casas, Conrado del Campo, José Iturbi, Alfred Cortot y Pierre Monteux. Fue catedrático de piano del Real Conservatorio Superior de Música de Madrid (1951-1974), y autor de un Ensayo sobre la técnica trascendente del piano (1944), y de La técnica del piano a través de todas las escuelas mundiales (beca de la Fundación "Juan March", 19591961). La obra dedicada a Pilar Bayona se titula Metamorfosis pianística sobre un tema de Ravel (piano) (1978). Fue también colaborador de Radio Nacional de España (desde 1944) y ejerció como crítico musical de los diarios Arriba y $A B C$.

31 [Antonio García Rubio], fue compositor, violinista y profesor del Conservatorio Superior de Música de Murcia. Premio "Fernández Caballero, 1955" de música, por su obra Cuarteto para arcos. Dedicó a Pilar Bayona su obra Praeludium, aunque se desconoce la fecha de su composición.

32 Director de orquesta alicantino, titular de la Orquesta Sinfónica de Madrid (1958) y catedrático del Real Conservatorio Superior ciudad aragonesa, en 1972, se celebró la II Semana de Música Española, para la que la Comisaría de la Música convocó un concurso de piano que llevaba su nombre: "Concurso Pilar Bayona”. Con tal motivo, realizó un concierto en homenaje a Manuel de Falla, dirigido por Rafael Frübeck de Burgos, en el que actuó como solista de las Noches en los Jardines de España ${ }^{33}$.

A continuación, todavía iba a participar en sendos conciertos en homenaje a Óscar Esplá (por el Club "Urbis” de Madrid), en 1974, y al poeta Vicente Aleixandre ${ }^{34}$ (por la New York University de Madrid), en 1978.

Finalmente, en 1979 realiza diversos conciertos en Zaragoza $^{35}$, y el 7 de noviembre ofrece su último recital de piano (fig.3), en la Caja de Ahorros de la Inmaculada ${ }^{36}$. El 13 de diciembre de 1979, Pilar Bayona murió a consecuencia de un atropello automovilístico.

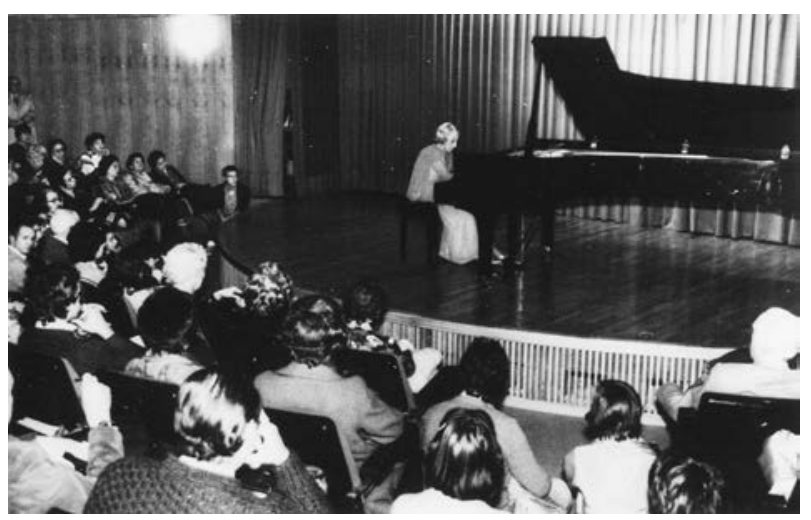

Fig. 3. Último recital de Pilar Bayona en 1979

de Música de Madrid. Dirigió la Orquesta Nacional de España y la Orquesta de la Radio Televisión Española.

33 Posteriormente repetiría esta misma obra en 1976, en aquella ocasión bajo la dirección de Ernesto Halffter $(1905 ; † 1989)$.

34 Vicente Aleixandre $(* 1898 ; \uparrow 1984)$-Premio Nobel de Literatura en 1977-, le envió un ejemplar de su libro Espadas como labios, en cuya dedicatoria expresaba su deseo de escucharla tocar, pues parece que no había tenido ocasión para ello en sus anteriores encuentros. Los escritores Ricardo Gullón Fernández (*1908; †1991) y Adolfo Lizón Gadea (*1919; †2011) le dedicaron también ejemplares de libros suyos.

35 En enero, actúa para la Caja de Ahorros de la Inmaculada, y en junio, para el Club "Calibo" y en el Colegio de Arquitectos de la ciudad, con ocasión de la clausura de la Muestra de Arte Aragonés.

36 En esas fechas, preparaba con ilusión un proyecto de veinte conciertos para estudiantes, bajo el patrocinio de la Fundación "Juan March". 


\section{Análisis de fragmentos}

\section{Soler, Sonata $\mathbf{N}^{0} 84$ en Re Mayor ${ }^{37}$}

He seleccionado un fragmento (fig.4) de esta sonata desde el comienzo hasta el final de la primera parte de la primera sección (compases 1-28).

14 Sonata No. 84 In D major
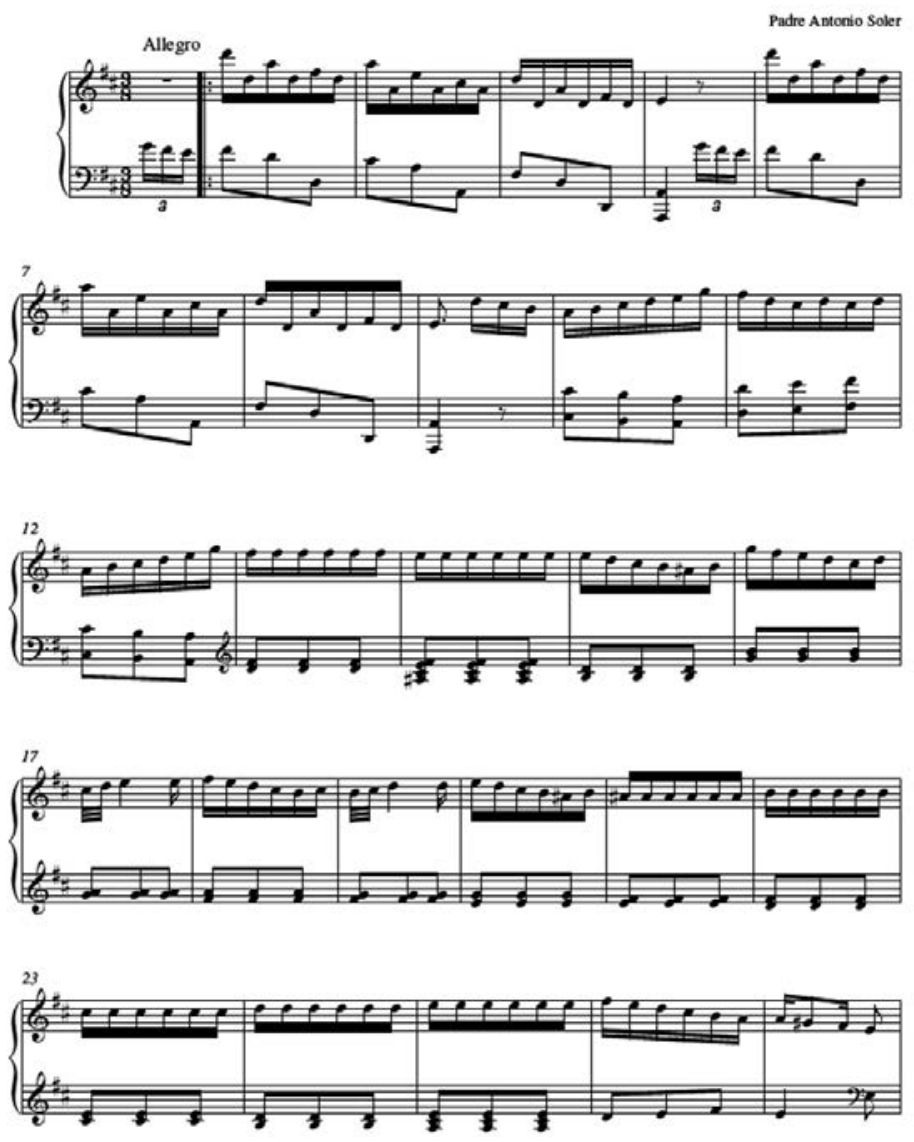

Fig. 4.

En primer lugar, de los datos que se derivan del espectrograma, se reporta una afinación de $429 \mathrm{~Hz}$, más baja que la de los $440 \mathrm{~Hz}$ habituales.

En el espectrograma global (fig.5) se observa una línea larga que aparece en la zona inferior de la imagen; corresponde a la frecuencia de la corriente eléctrica de 50 hercios
- la frecuencia corresponde a un sonido alrededor del sol sostenido - . Esta línea aparecerá en otras ocasiones, cuando las grabaciones son antiguas, o de mala calidad. El problema de esta línea, es que enmascara la imagen de otros sonidos alrededor de esa frecuencia; en este caso el $L a$, precisamente la dominante de la tonalidad de la obra ahora en cuestión.

37 El número 84 de esta Sonata corresponde a la edición de Samuel Rubio. Pilar Bayona utiliza la edición de Sonatas de Joaquín. Nin. La grabación, es una grabación no profesional, realizada con un aparato casero. Corresponde a una reunión en casa de los Urgoiti, - amigos de Pilar Bayona-, en Junio de 1970. El soporte guardado en el archivo es una cassette. Existe otra grabación de este tipo correspondiente al bis de un concierto pero la calidad es peor que esta. Yo he utilizado como referencia la edición SOLER (2006). 


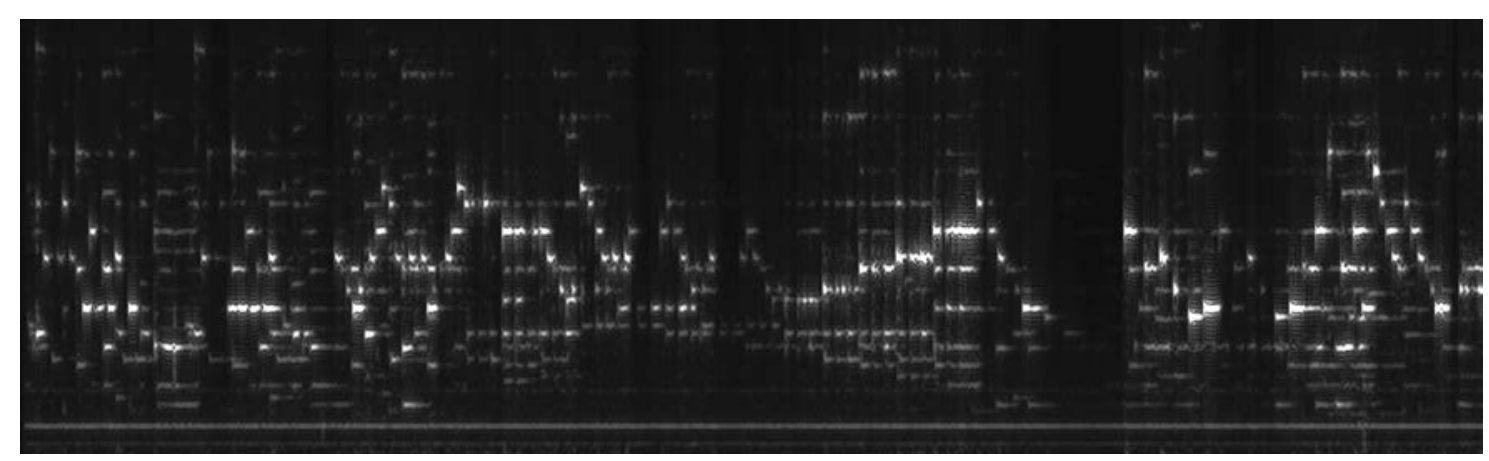

Fig. 5. Espectrograma global de todo el fragmento.

A lo largo de todo el fragmento, se observa que, muchos de los sonidos, no presentan la forma de embudo típica de un sonido con resonancia en el piano, más bien aparecen en forma de cuadrado (fig.6). Ello indica sonidos cortos incluso picados, muy sueltos, pero además, es un síntoma de que ha puesto muy poco pedal, o casi ninguno.

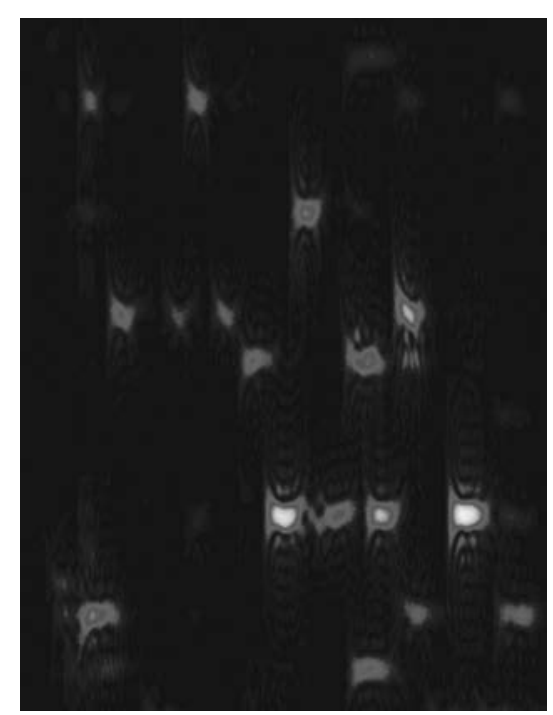

Fig. 6. Los sonidos presentan una forma cuadrada.

Estos sonidos "cuadrados" aparecen ya, nada más comenzar, en las voces intermedias de las primeras figuraciones y además, con unas formas picudas que se diferencian de los ataques un poco más planos y largos de las notas agudas del tema inicial; esto quiere decir que los sonidos alargados están más apoyados con el dedo. En la imagen en 3D, se observan estos sonidos cortos, más destacados que los de la parte aguda. Esto viene a demostrar que la duración del sonido tiene más influencia en los planos sonoros de lo que pueda parecer, desde mi punto de vista, puesto que en la escucha se percibe claramente la voz superior en primer plano (fig.7). A esto habría que añadir que el oído tiene más sensibilidad en esa región (alrededor de los $1000 \mathrm{~Hz}$ ), y por tanto, se perciben los sonidos más ampliados, con más nitidez.

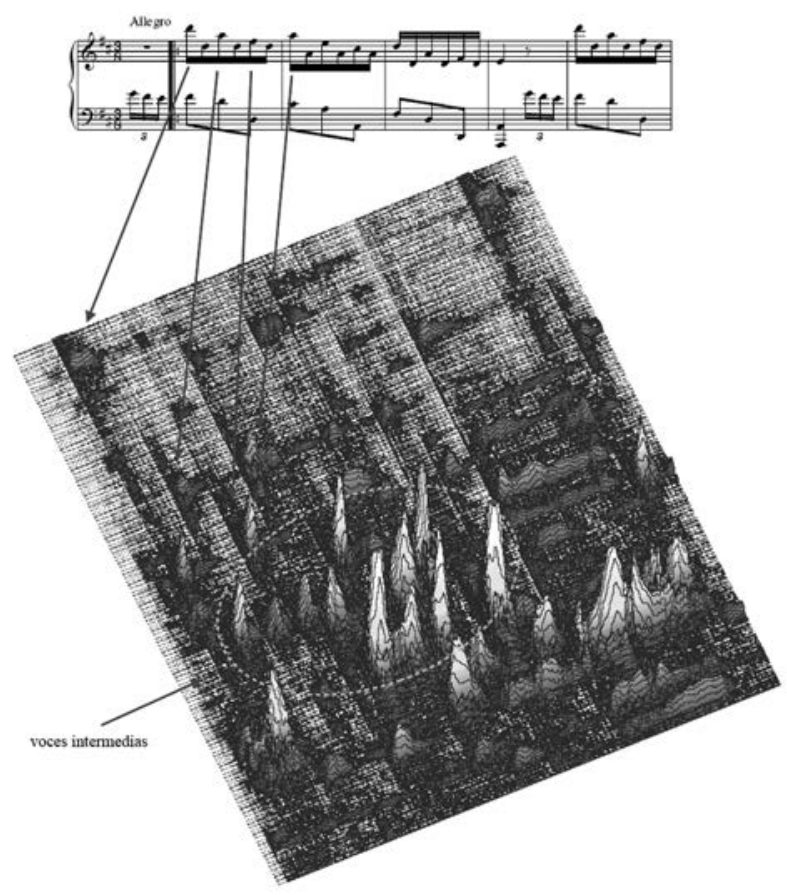

Fig. 7.

Resulta de mucha expresividad el interés que la pianista le da a las apoyaturas de las fusas de los compases $17 \mathrm{y}$ 19 (figuras 8 a y 8 b). Sin necesidad de medir, se ve a simple vista como ha realizado los diseños. Por un lado, estira la primera corchea de las tres de la mano izquierda, apoyando 
y dando más peso a la parte fuerte, y por otro lado, retrasa la entrada de la primera nota las tres fusas; el resultado es de mucho efecto expresivo y más sumado al diminuendo gradual que va a preparar el final de la frase.

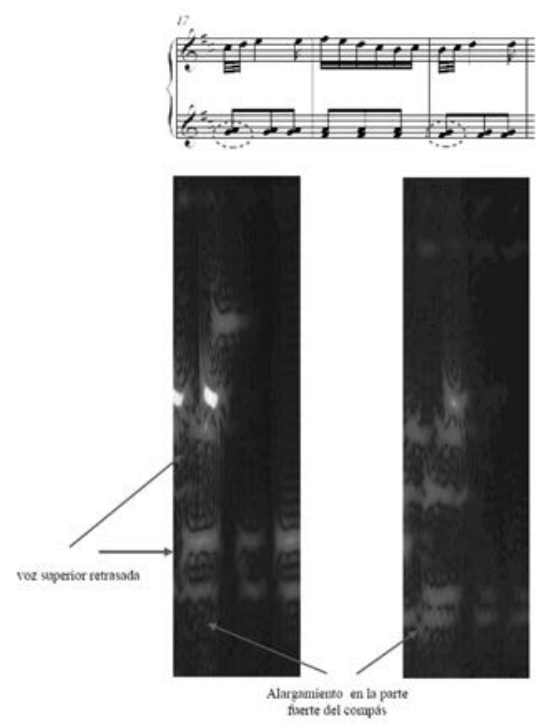

Fig. $8 a$

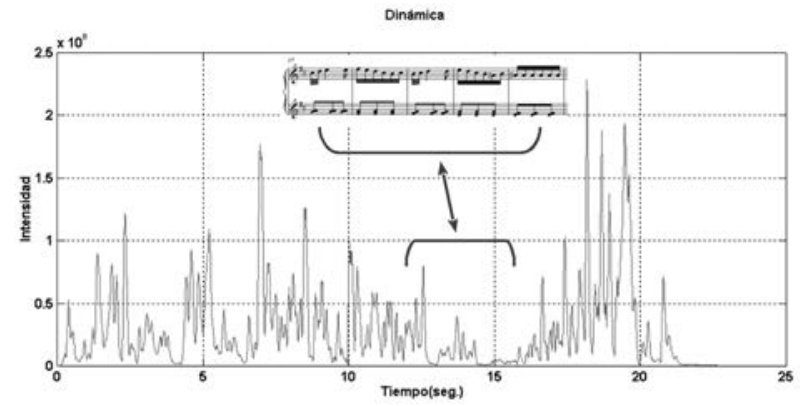

Fig. $8 b$.

A pesar de la baja calidad de la grabación, se puede apreciar la claridad de planos sonoros de la mano derecha y la mano izquierda. También se puede observar, el crescendo gradual hacia el $F a$ del final de la frase (fig. 9). La pianista, sin embargo, baja la dinámica precisamente en esta nota, el punto culminante, aun tratándose de la parte fuerte del compás. Éste efecto se repite, de alguna manera, en el compás siguiente, en el que la pianista le da más interés a la anacrusa, que a la parte fuerte del compás antes de terminar, aun siendo no sólo parte fuerte del compás, sino apoyatura.

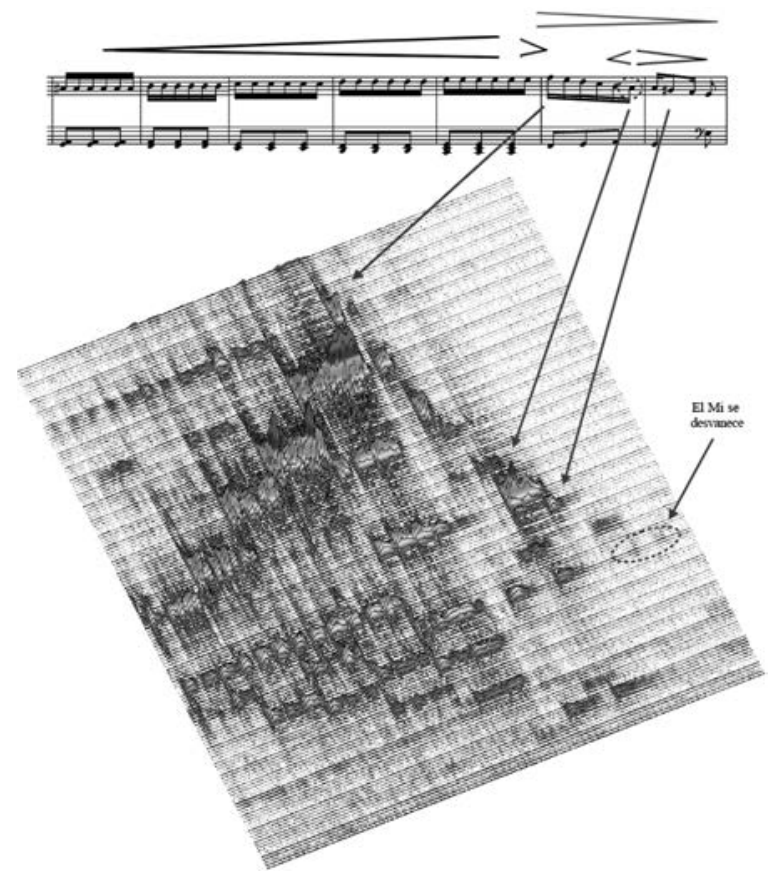

Fig. 9.

Sin embargo el efecto global hasta el final de la frase es de un gran crescendo y diminuendo, aunque en el detalle sea un poco diferente, como también se observa en la gráfica de la dinámica anterior.
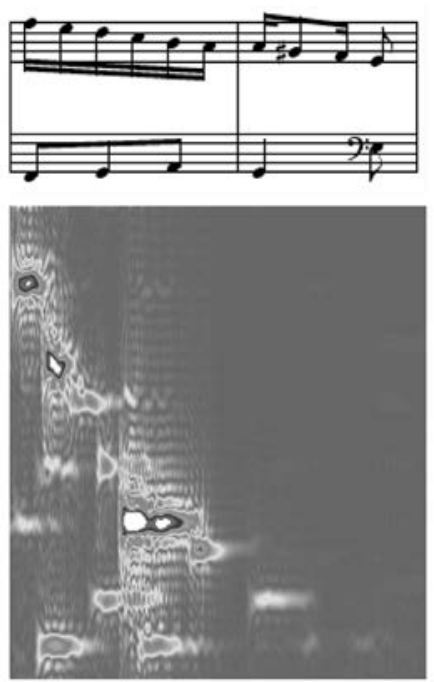

Fig. 10. Ritardando final de frase. Detalles de la dinámica, nota a nota. 
Especialmente interesante me parece como ha realizado Pilar Bayona todo el ritardando del final de esta sección (fig. 10). De los primeros cinco primeros compases, donde se inicia el crescendo final, sólo he medido la primera nota del compás, tomando las medidas de la semicorcheas de los dos últimos compases.

El tempo metronómico medido de este final es T.M.= 242.2, la semicorchea (negra con puntillo 40.37). El movimiento de la frase parece mostrarnos como primer punto culminante el momento que ha terminado el cresendo, es decir en el $F a$. Se observa que el movimiento de esos cinco compases tienen la misma forma que el movimiento total de los compases de todo el pasaje. En realidad, ese movimiento parece querer llegar a otro escalón superior en que las notas van más lentas para seguir haciendo otro ritardando al final.

Si se analizan los valores de la frase aparecen los cinco compases anteriores acelerados paralelamente al crescendo, aunque la sensación es que el compás de la semicorcheas realmente ha cambiado de tempo.

Desde mi punto de vista la gráfica del movimiento por compases muestra un gradual ritardando de lo más elegante, pero, por otro lado, creo que muestra una gran habilidad de la pianista para integrar el detalle en la forma más grande (fig. 11).
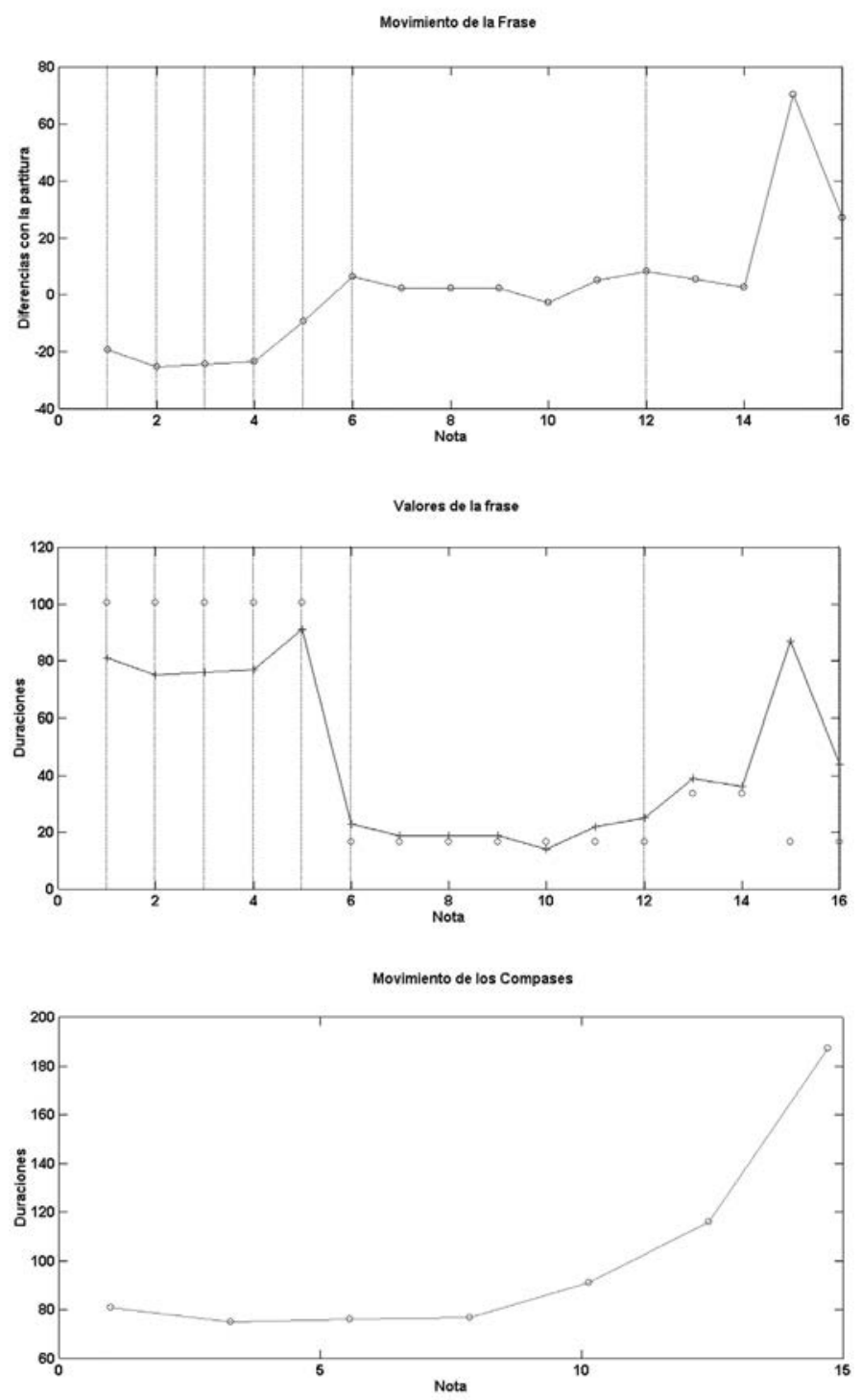

Fig. 11. 


\section{Albéniz, Lavapiés (Tercer cuaderno de Iberia) $)^{38}$}

He seleccionado un fragmento (fig.12) de "Lavapiés" de Iberia, por el carácter castizo puramente madrileño que le imprime la pianista, personal y subjetivamente una de las mejores versiones que he escuchado nunca. Muestro aquí unas líneas del tema central de la segunda sección de la obra.
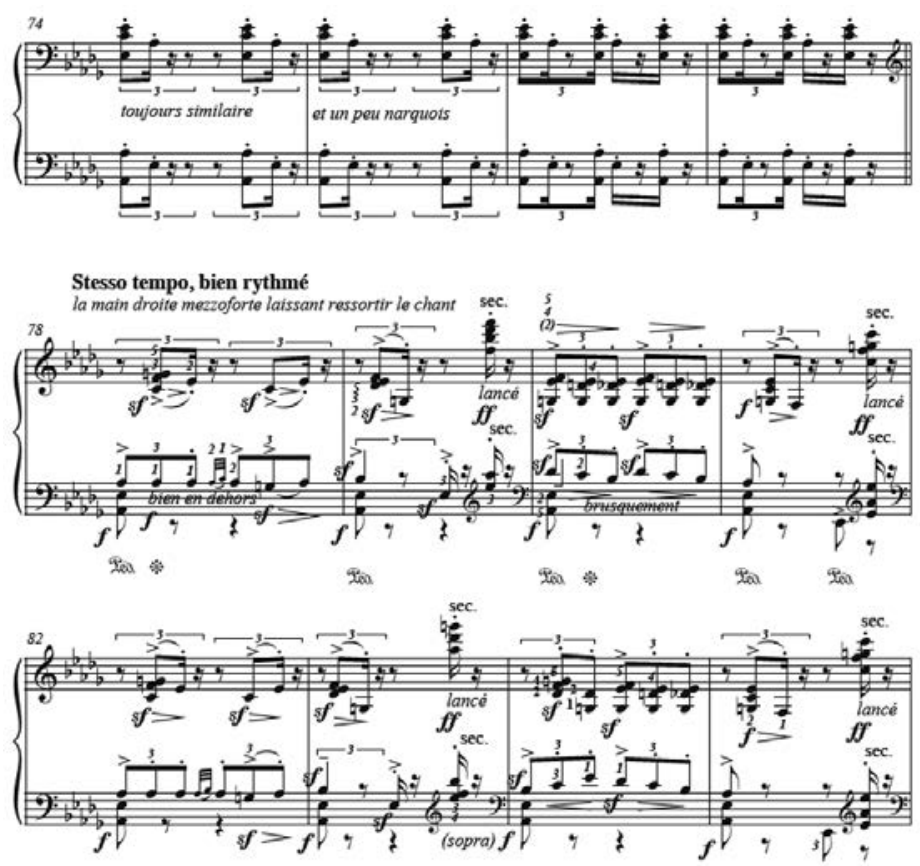

Q

Soa

To Tai

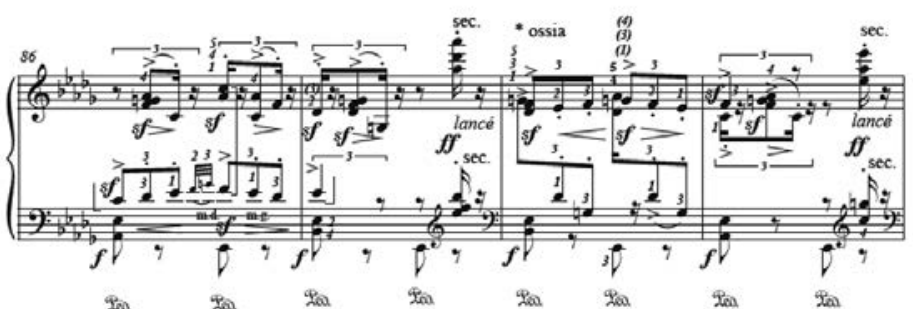

Fig. 12.

Respecto a los acordes del principio, que sirven de introducción al tema de esta sección de la obra, compases 70-77, hay que destacar, en primer lugar, la forma de los acordes, que en general es muy regular y equilibrada, como muestra el espectrograma (fig.13), con todos los armónicos de los acordes. 


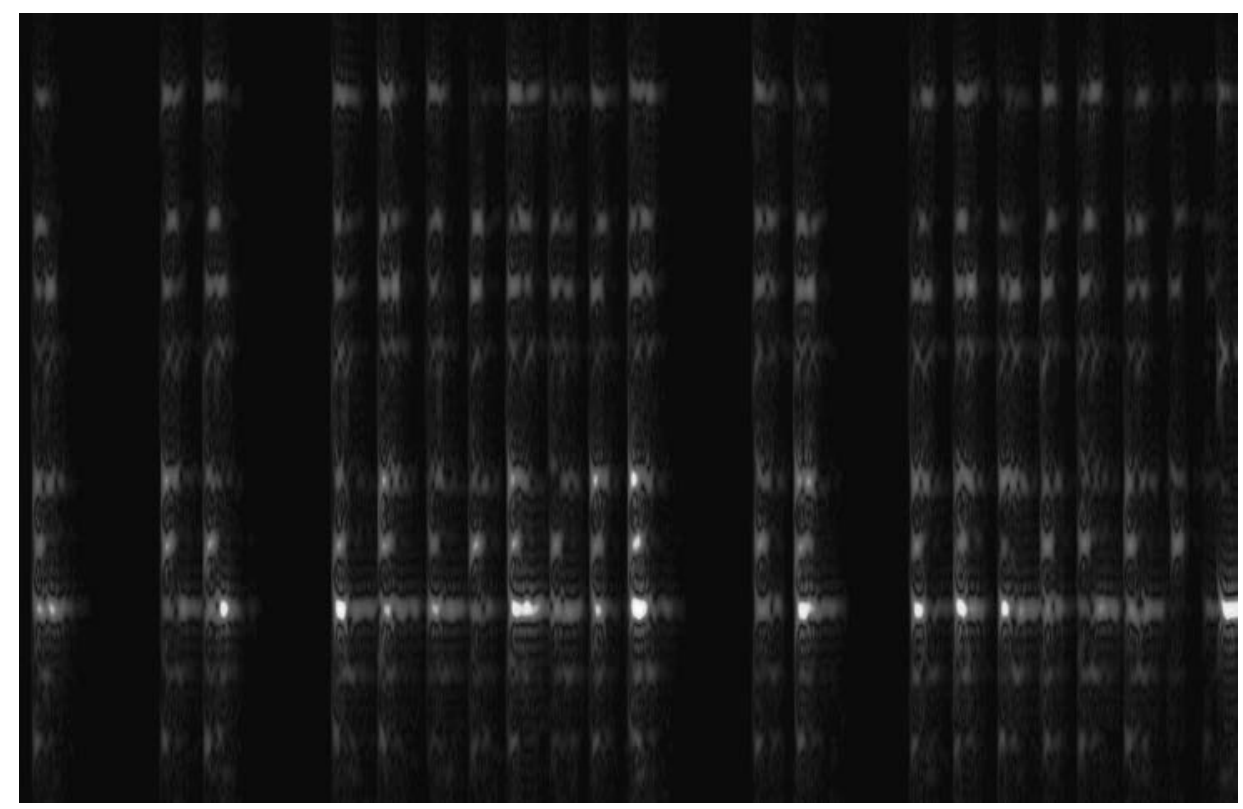

Fig. 13.

Observando con más detalle la estructura del acorde inicial, idéntico a cómo va a realizar todos los demás, se advierte el empaste de todas las notas que lo componen.

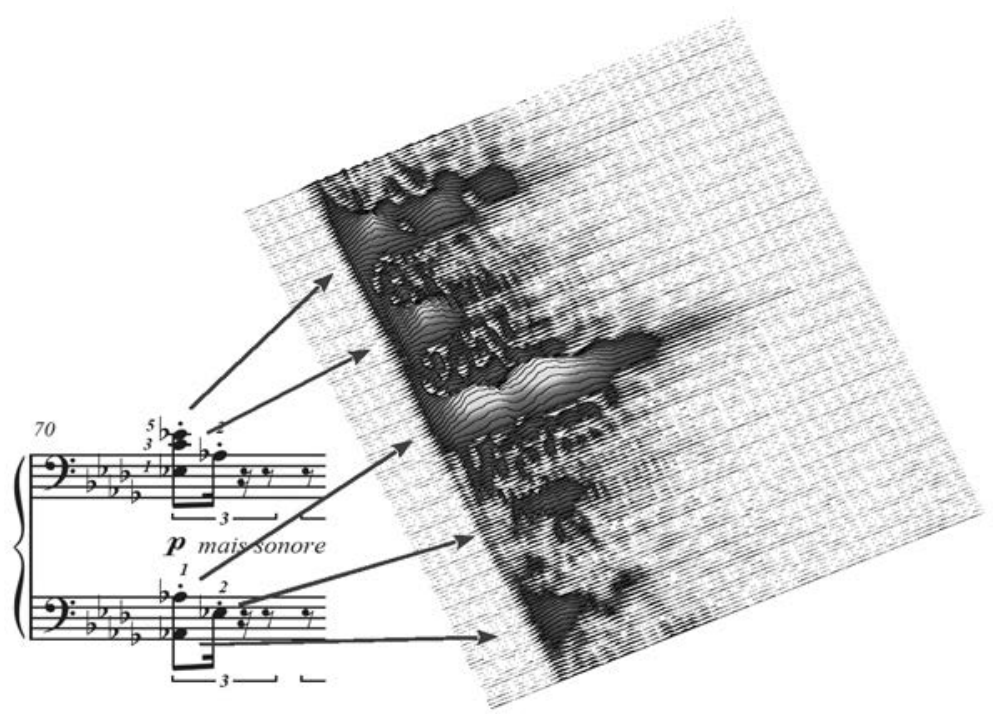

Fig. 14.

Se puede ver que el dedo pulgar en el La bemol se queda un poco pegado (fig.14), y algunas veces más intenso, como aparece en la imagen global del espectrograma pero, por otro lado, al ser la fundamental del acorde, pienso que contribuye a esa fusión de las notas componentes del acorde.
Pilar Bayona, realiza una personal caracterización rítmica de este pasaje. He medido la siguiente gráfica (fig. 15), tomando valores en el momento del ataque del acorde, sin incluir la segunda nota que le acompaña, pues me ha parecido lo distintivo del fragmento. 


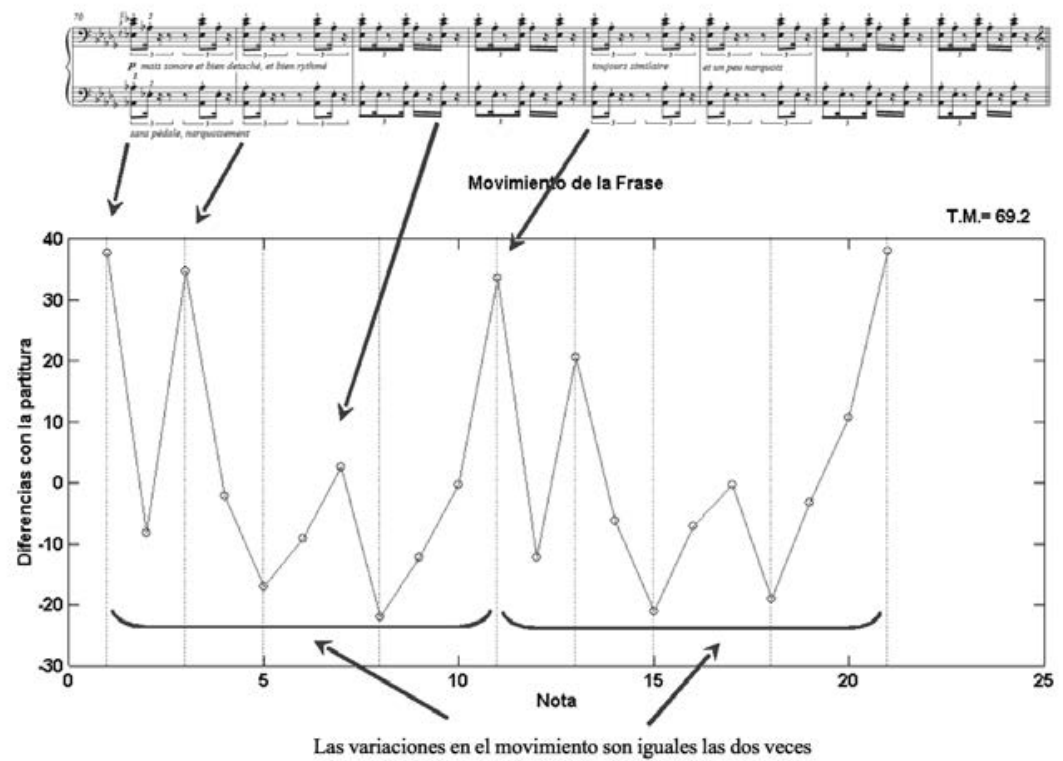

Fig. 15.

En primer lugar, se observa que en las dos repeticiones (compases 70-73 y 74-77) la gráfica es prácticamente idéntica, mostrando un perfecto control rítmico. Así, la realización de la pianista, muestra los alargamientos en los dos primeros compases (y su repetición), más sujetados, mientras que en los otros dos compases, el movimiento es más rápido. Comparando esta información con el espectrograma, el estiramiento de los dos primeros compases y su repetición, se deben al alargamiento de los silencios entre ataques de los acordes, pues en la gráfica del movimiento de la frase, el último valor del compás está acortado (fig. 16).

Por otro lado en las dos subdivisiones de la frase los dos elementos tienen una realización diferente. La pianista vuelve a sujetar el tiempo en las partes débiles del compás. La gráfica por compases corrobora también este sentido rítmico, dos compases más lentos, dos compases más movidos. El ritardando de final de frase es muy leve, uniendo esta introducción con la siguiente frase, que es el motivo central de la obra.

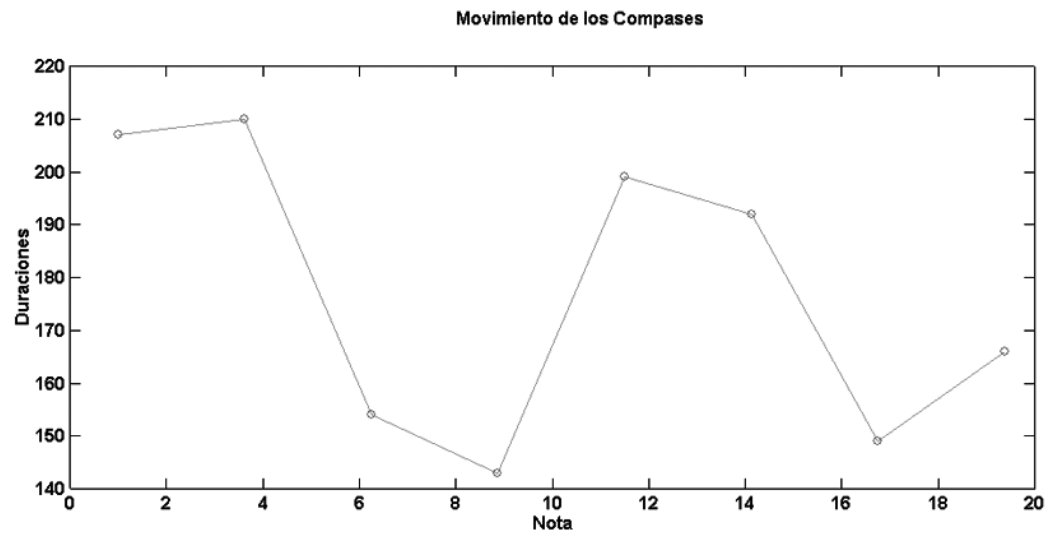

Fig. 16. 
A continuación de esta pequeña introducción aparece el tema principal. Se observa, en primer lugar, que el acorde de inicio de cada elemento de esta frase está roto. La pianista separa las dos notas graves del acorde de la nota principal del tema, se supone que para colocar éste en primer plano (figuras 17a y 17b).

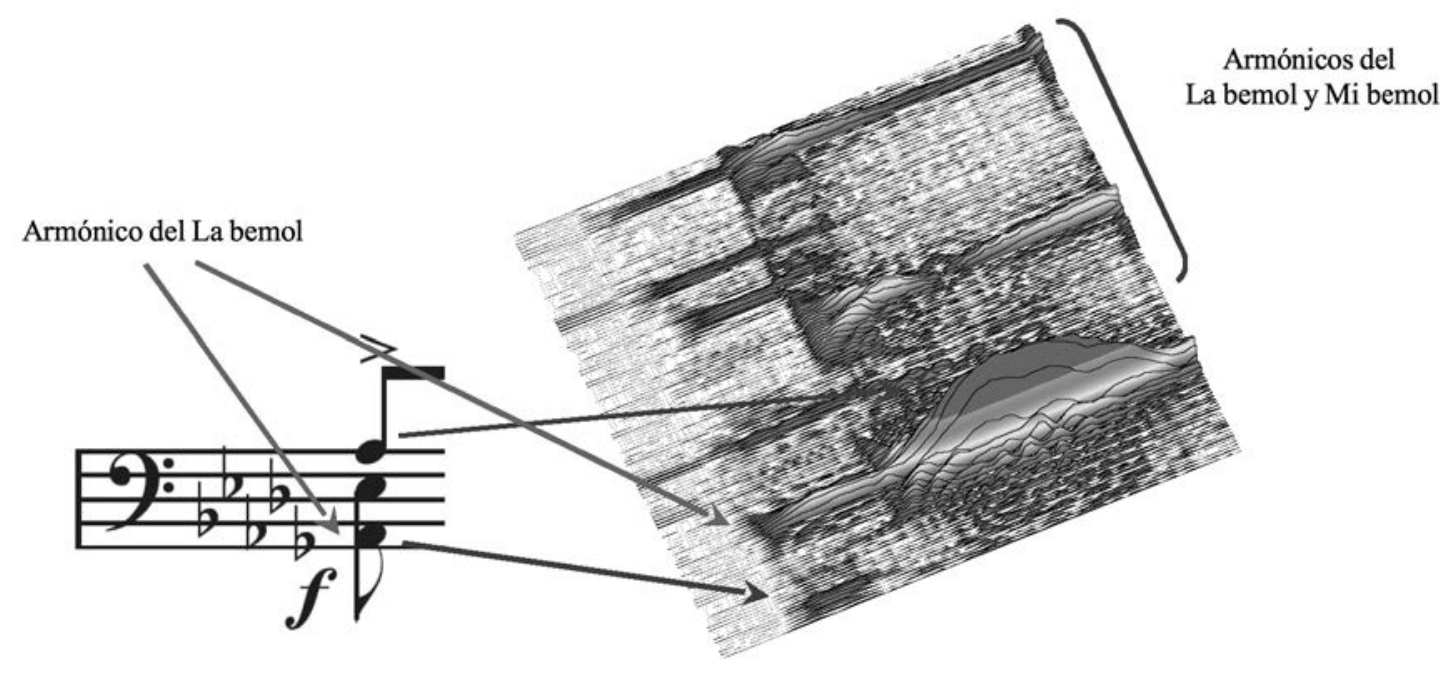

Fig. 17a.

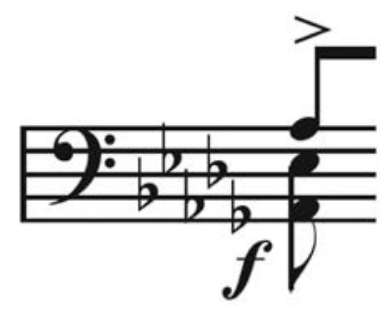

Albéniz

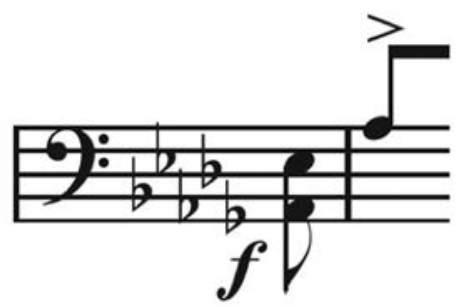

\section{Bayona}

Fig. 17b.

Esto también se puede observar en la imagen global del espectrograma, donde aparecen numerosos acordes, en el inicio de cada compás, quebrados (fig. 18). 

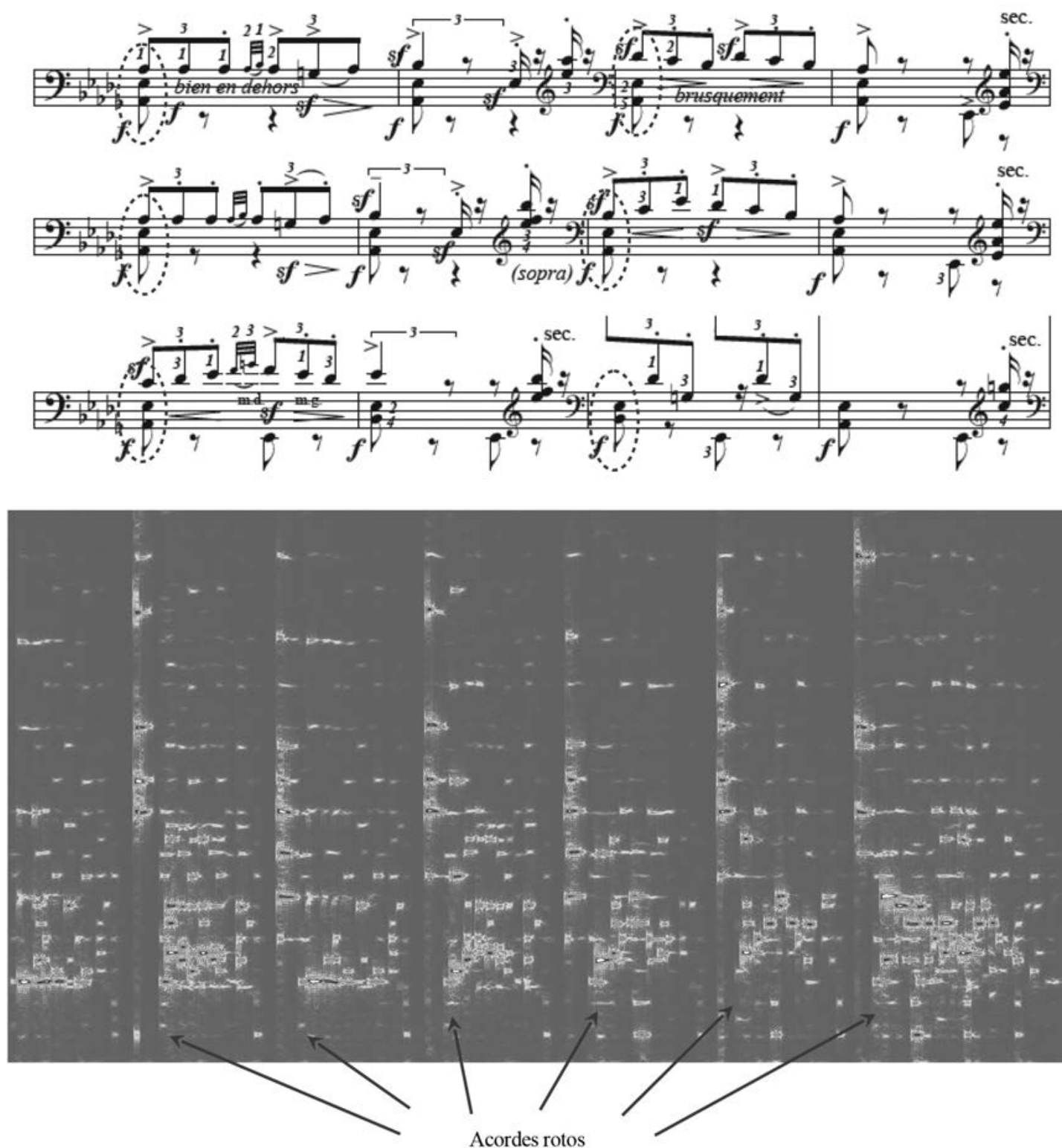

Fig. 18.

Personalmente pienso que, aparte de servir para dirigir la atención al oyente hacía el tema principal - y además facilita la ejecución técnica, pues Pilar Bayona tenía la mano pequeña-, contribuye a dar más fuerza y carácter al pasaje. En una primera escucha, los acordes quebrados no se perciben, entre otras razones porque los planos de sonido que realiza la pianista están muy bien delimitados.
Se pueden encontrar otras modificaciones más importantes de la obra en la versión de la pianista. Analizando con un poco de detalle, se observa que añade algunas notas que no están en la partitura, como por ejemplo un Si bemol en el compás 79 , que se vuelve a repetir en el compás 83 , o un Mi bemol añadido con una octava, que en el espectrograma y en la escucha, se percibe en los compases 81, 85 87, 89, y 93 (fig. 19). 


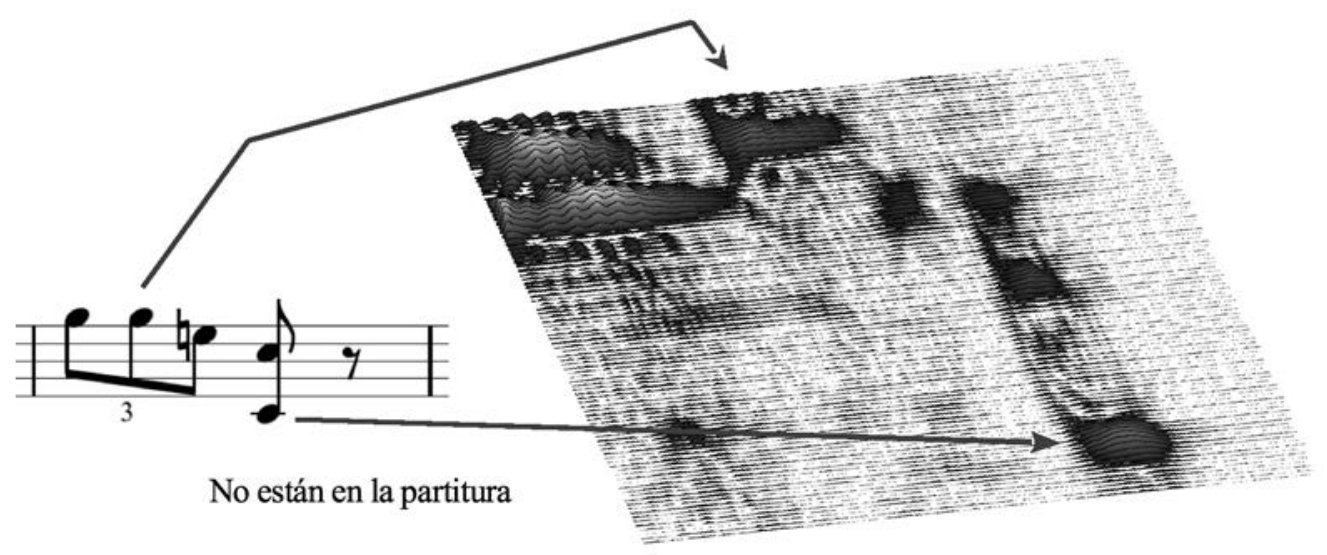

Fig. 19.

Se pueden resumir esquemáticamente estas modificaciones en estos dos elementos (fig. 20):

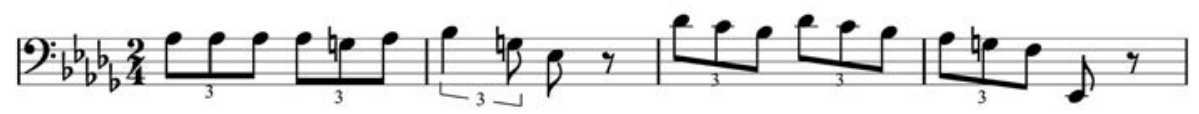

Albéniz

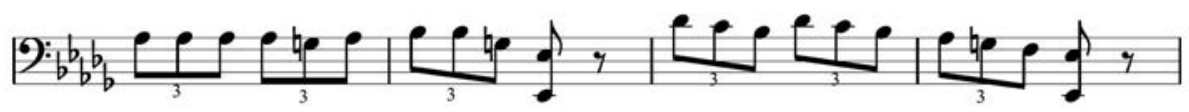

Bayona

Fig. 20.

Desde mi punto de vista son dos modificaciones importantes que realiza la pianista porque corresponden a notas principales de la melodía, y no a notas en segundo plano. Mi opinión es que su intención es hacer más explícito lo que está implícito, con la finalidad de dar más claridad al tema del pasaje.

Debido a la tesitura en la que se encuentra el tema, ha sido necesario realizar un espectrograma un poco diferente a los anteriores, con una ventana de análisis más pequeña para poder desplazarla a lo largo de la señal. Esto da un poco más de resolución temporal y permite medir un poco mejor los ataques. A veces ha sido difícil precisar en el espectrograma los momentos de los ataques, por eso he tenido que medir los armónicos donde los ataques están más claros.

Los planos sonoros también están relativamente definidos En un vistazo general, ya se puede ver en primer plano la melodía principal. Al igual que se percibe en la primera escucha, cada uno de los diseños del tema los realiza en $d i$ minuendo, con lo cual la dinámica de este pasaje está clara. La envolvente de amplitud (fig. 21) lo muestra igualmente: 


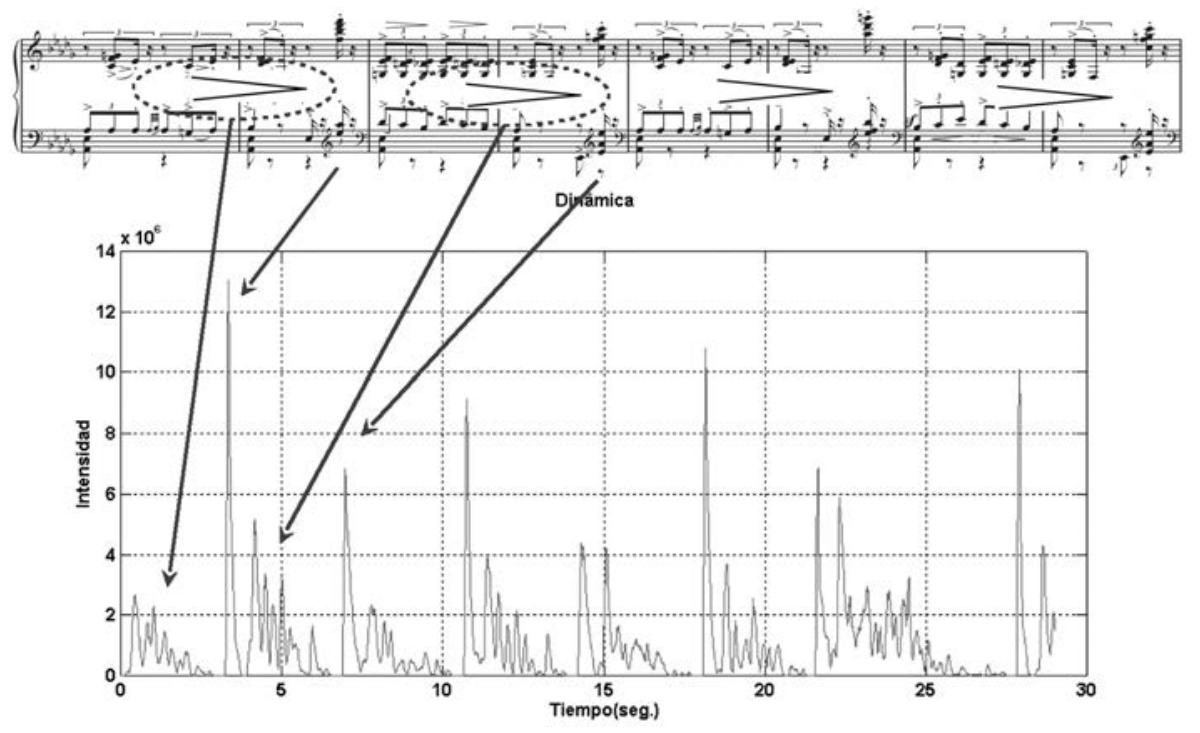

Fig. 21.

Interesante observar también la relación que hay entre los acordes en sforzando en la zona más aguda, al final de cada dos compases, que están organizados de la manera más- menos-mas-menos es decir, más sonido o menos sonido, pero dentro de un ataque fuerte súbito (figuras 22a y $22 \mathrm{~b}$.

En toda esta serie de diminuendos en los motivos de este pasaje, se puede encontrar otro detalle respecto de la dinámica.

Me refiero a un diminuendo en pequeñas células o partes del motivo, cuando estos elementos son ascendentes, y que aparecen en diferentes lugares.

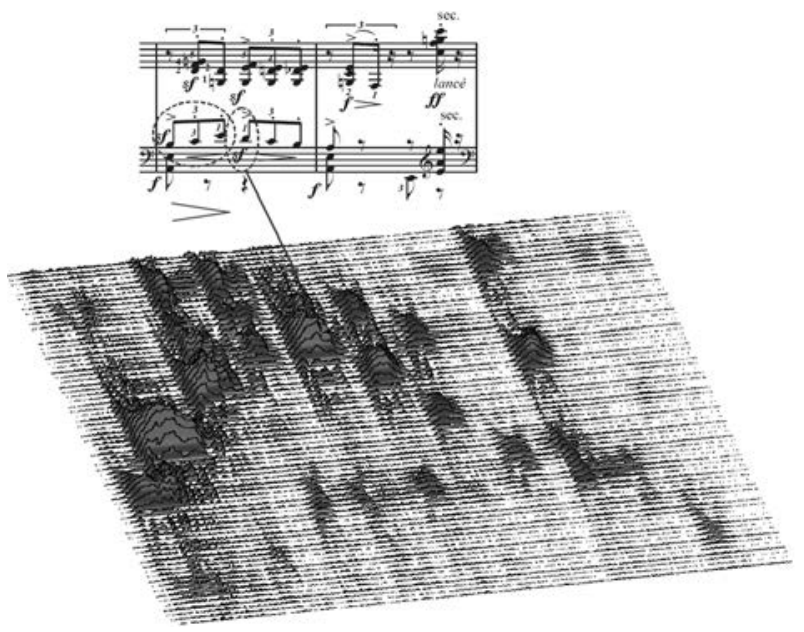

Fig. $22 a$.

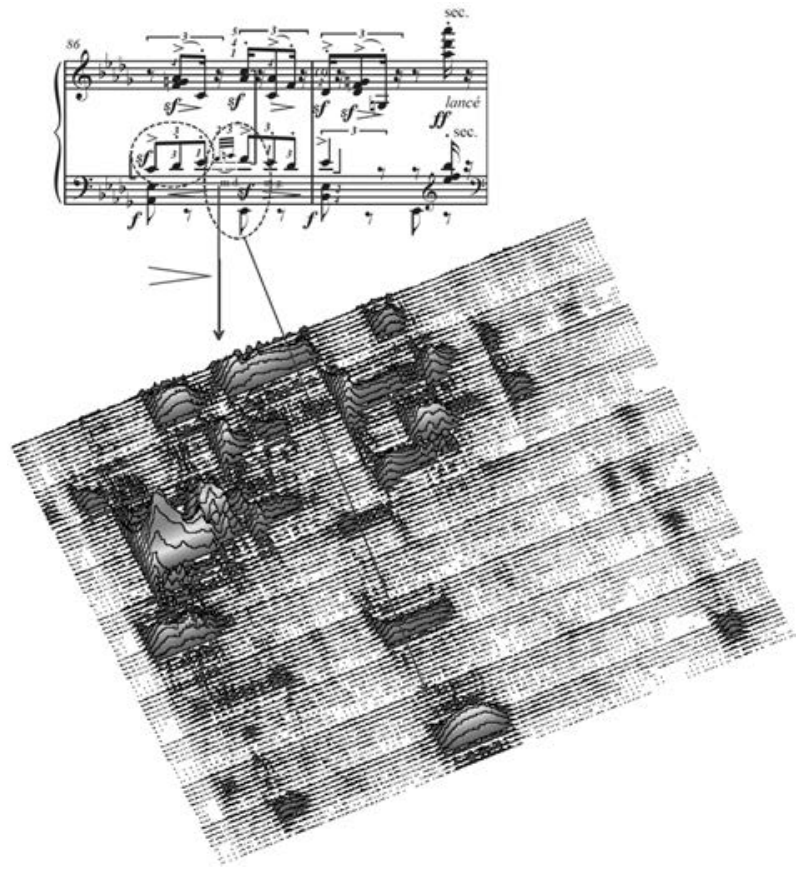

Fig. $22 b$.

Resumiendo, se puede decir que en el aspecto dinámico, la pianista inicia cada motivo con un impulso decidido, que cae en un primer momento, independientemente de la dirección que tomen las notas y cierra con suavidad el final. 
En el segundo caso se observa además como ha ejecutado las notas de adorno "antes" del bajo, en la subdivisión fuerte del compás.

Me ha parecido interesante ver cómo articula y realiza el movimiento de las corcheas. Puesto que los diseños son muy similares y la partitura un poco farragosa, he seleccionado los cuatro primeros motivos (8 compases) para ver con más claridad las gráficas.

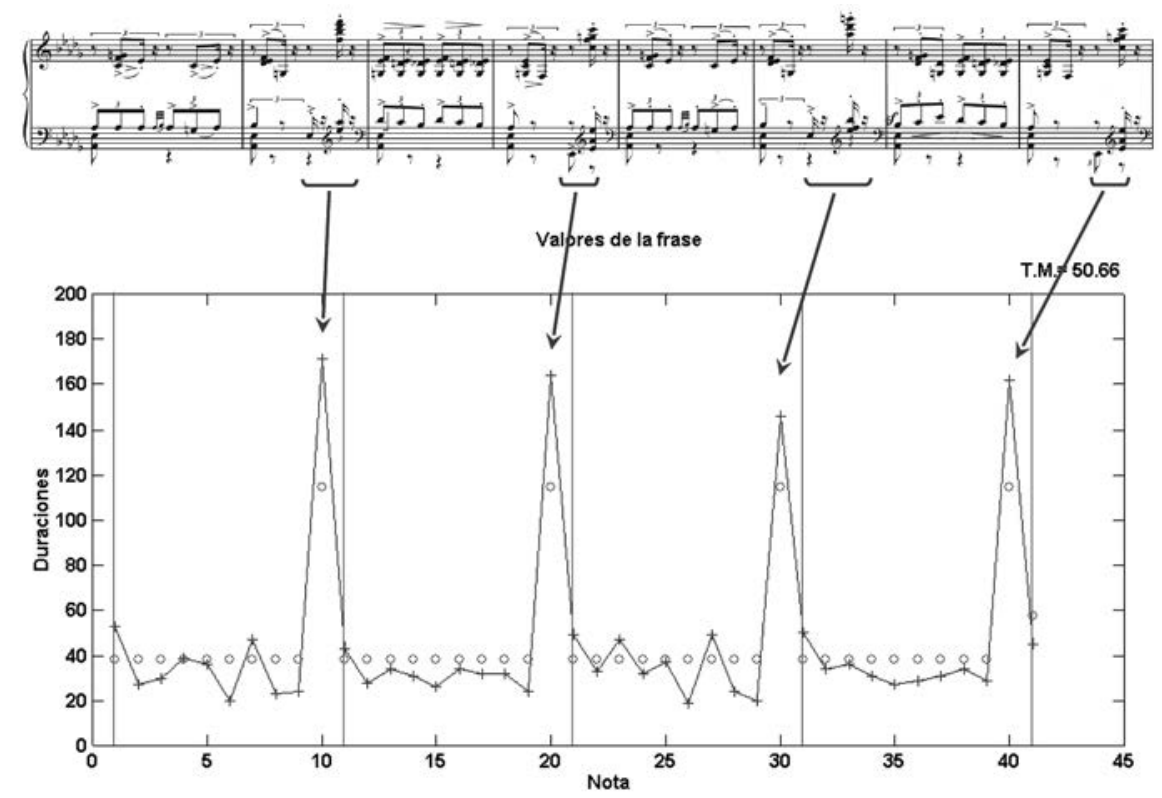

Fig. 23.

En la gráfica de valores (fig. 23) se advierte dos movimientos diferentes; por un lado están los notables estiramientos en los acordes sforzándo - he integrado la nota anterior, Mi bemol, con el acorde en la medida - en la parte débil del compás, y por otro, los tresillos de corcheas, que están más movidos, más acelerados pero con diferentes maneras. Para observar ello con más detalle, utilizo la gráfica del movimiento de la frase (fig. 24). 


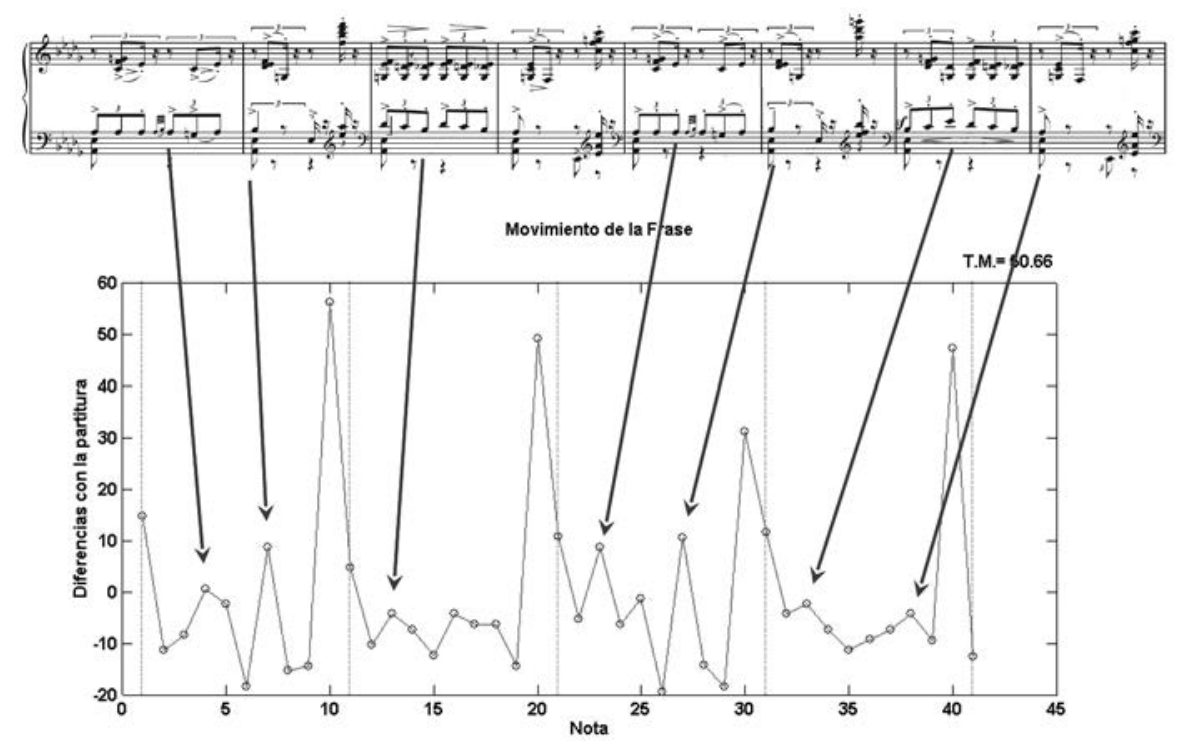

Fig. 24.

Se puede observar cuáles han sido los valores más exagerados y los pequeños picos que aparecen en puntos muy concretos. En general las variaciones han sido muy pequeñas; el mayor estiramiento corresponde a los acordes de las partes débiles y después aparece en las partes fuertes del compás. Se observa que es característico que el final de cada motivo termina en un ligero acellerando. Los demás picos podrían interpretarse con unas indicaciones de articulación, con las que podría escribir el fragmento de la siguiente manera (fig. 25), teniendo en cuenta que estas son muy ligeras.

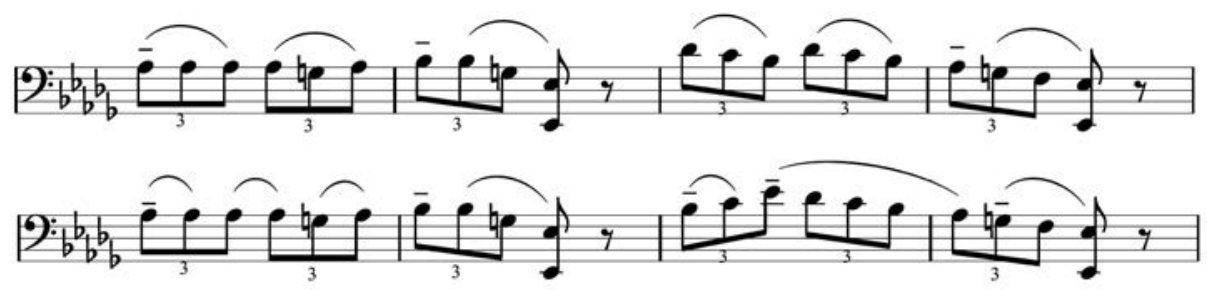

Fig. 25.

La barra encima de las notas significa que son ligeramente más largas; las ligaduras la agrupación de notas en función del dibujo. 
Albéniz, Eritaña (Cuarto cuaderno de Iberia) ${ }^{39}$

Es conocida entre los pianistas profesionales ${ }^{40}$ la singular caracterización que hacía Pilar Bayona del comienzo de Eritaña de la Suite Iberia de Albéniz, por ello he seleccionado este fragmento para realizar un análisis (fig.26).

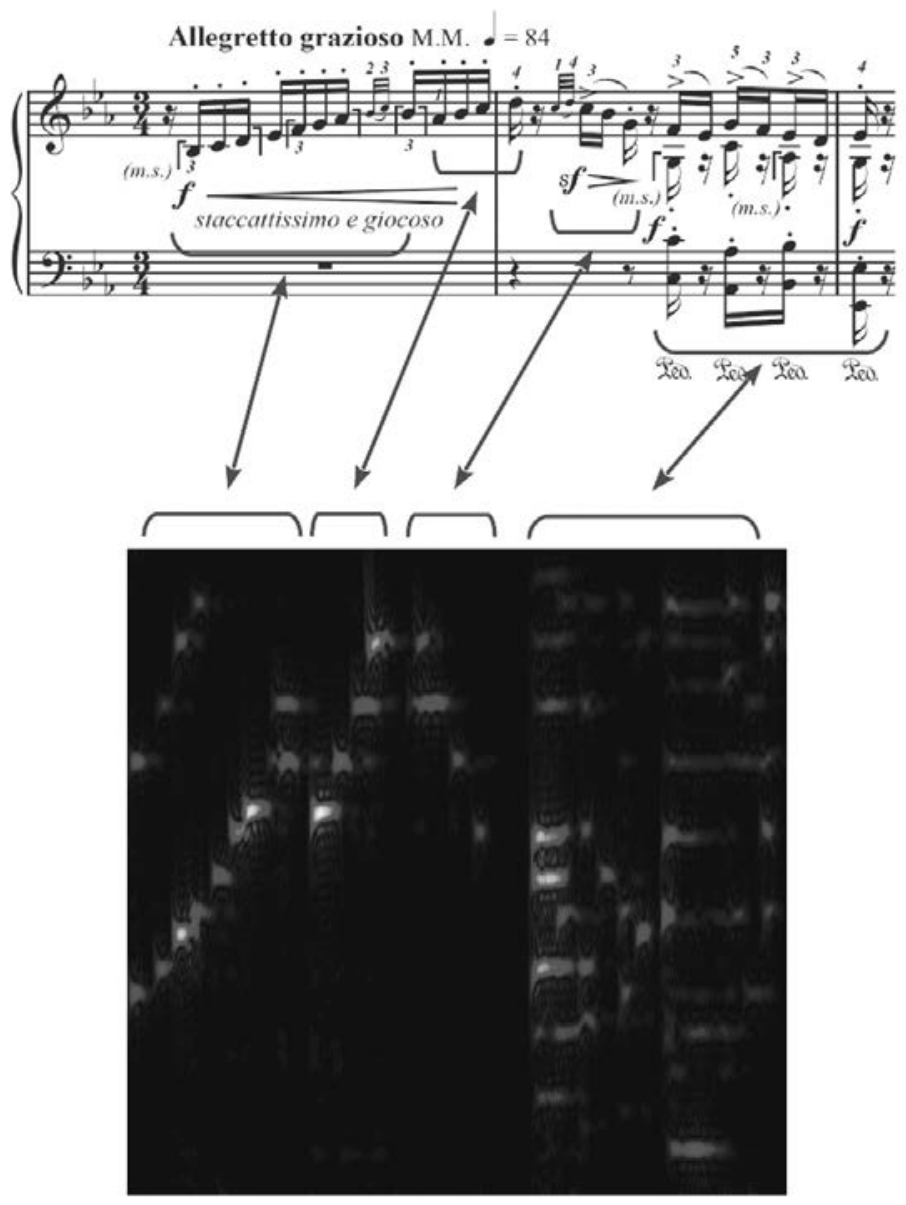

Fig. 26.

39 La grabación corresponde a BAYONA (1955). La edición de la partitura utilizada en este trabajo es ALBÉNIZ (2014). (Audio 37).

40 El pianista Pedro Carboné comenta sobre este comienzo: [...] "También recibí otra lección póstuma gracias a una grabación de Eritaña, pieza que nunca le había oído tocar en directo. Pero que desparpajo en el primer compás, nadie puede tocar eso así más que ella.” Véase: VARIOS AUTORES (2009): 46. 


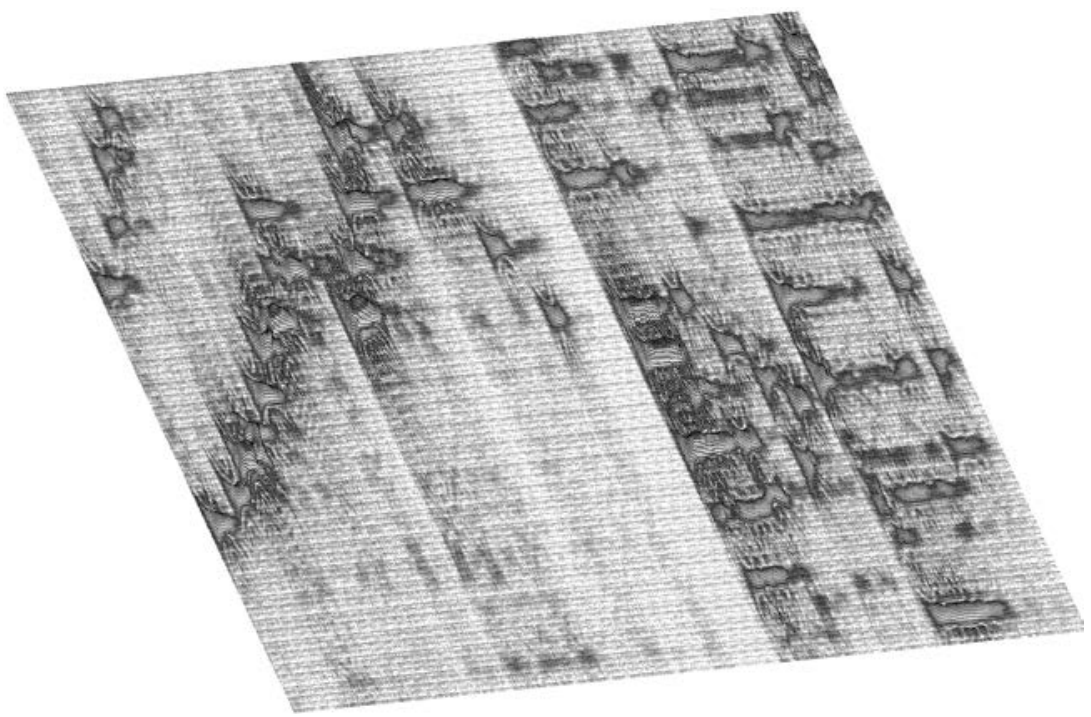

Fig. 27.

En la imagen tridimensional (fig. 27 y 28 ) se observa la importancia de los dos La bemol, especialmente del segundo, por la separación del sonido que le precede, la intensidad con que lo ataca y el diminuendo de los sonidos posteriores.

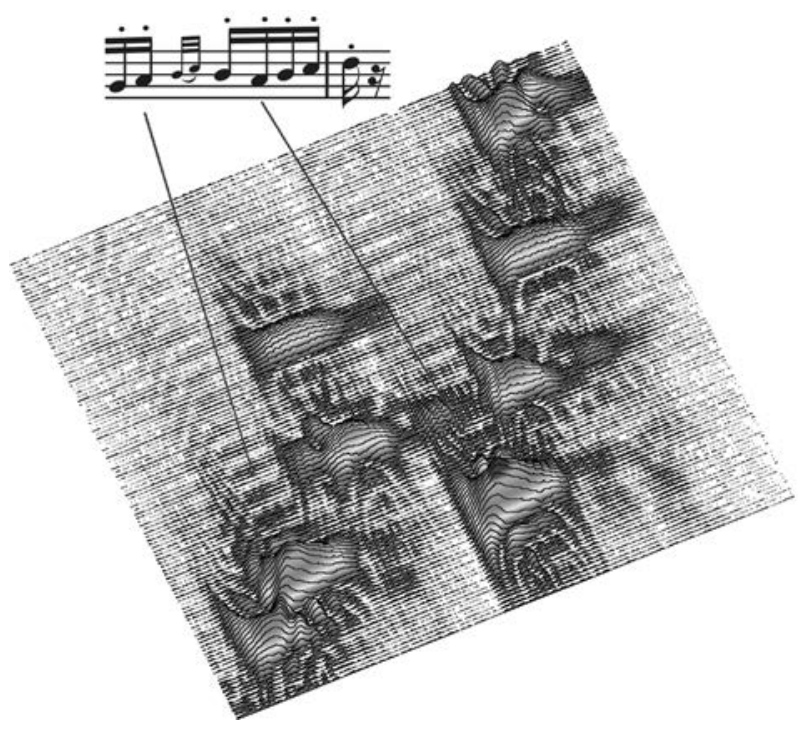

Fig. 28
Por tanto, el diminuendo que indica la partitura el compositor en el compás siguiente, lo inicia antes, aunque sigue conservando la relación dinámica de las tres últimas notas que empiezan con un sforzando (círculo azul), según marca la partitura, pero con una articulación muy suelta (los sonidos aparecen muy cortados); ello sugiere también muy poco o nada de uso del pedal. El regulador inicial lo finaliza en el primer La bemol.

Un aspecto interesante de analizar es la ejecución de los dos adornos (fig. 29). El primero está ejecutado, levemente, con mayor intensidad que el segundo, y con una brevísima respiración, pero en ambos el mayor apoyo se dirige a la nota real. La brevedad de la nota auxiliar en el $2^{\circ}$ mordente se percibe en la audición como con mayor perfección todavía. Esto sería una prueba de que lo que sucede después de atacar una nota en la tecla, si que tiene importancia en la propia calidad y claridad del sonido. 


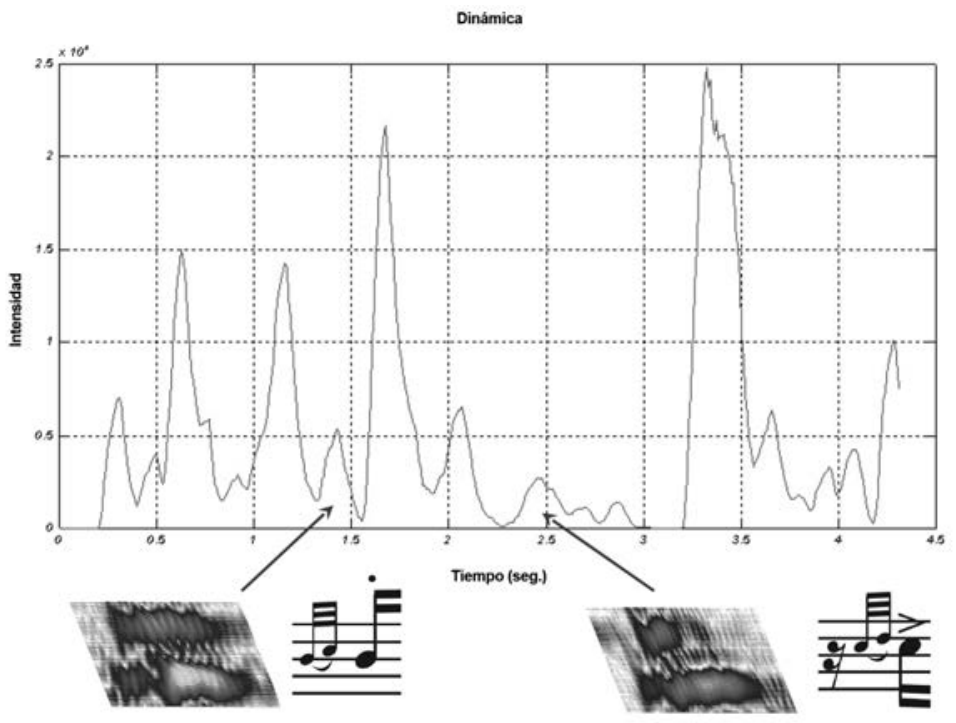

Fig. 29.

Sobre la agógica del fragmento (fig. 30). El tempo metronómico medido da un valor de negra de 86,5. El gráfico inferior con las variaciones de tempo nota a nota, muestra tres picos, representa los puntos de interés donde ha retrasado el tempo; se puede observar que delimita cuatro espacios. Estos picos se producen justo antes de los dos adornos (marcados con círculos), lo cual sugiere una respiración como preparación antes de atacar los adornos. La pianista, seguidamente, acelera las notas posteriores y antes del ataque de los acordes, esta vez, el alargamiento es un poco más largo, para terminar en el tempo inicial, de manera conclusiva. Al contrario de lo que se pudiera pensar, las apoyaturas de los acordes, están aceleradas (la gráfica muestra breve-larga, o iguales) en vez de alargadas.

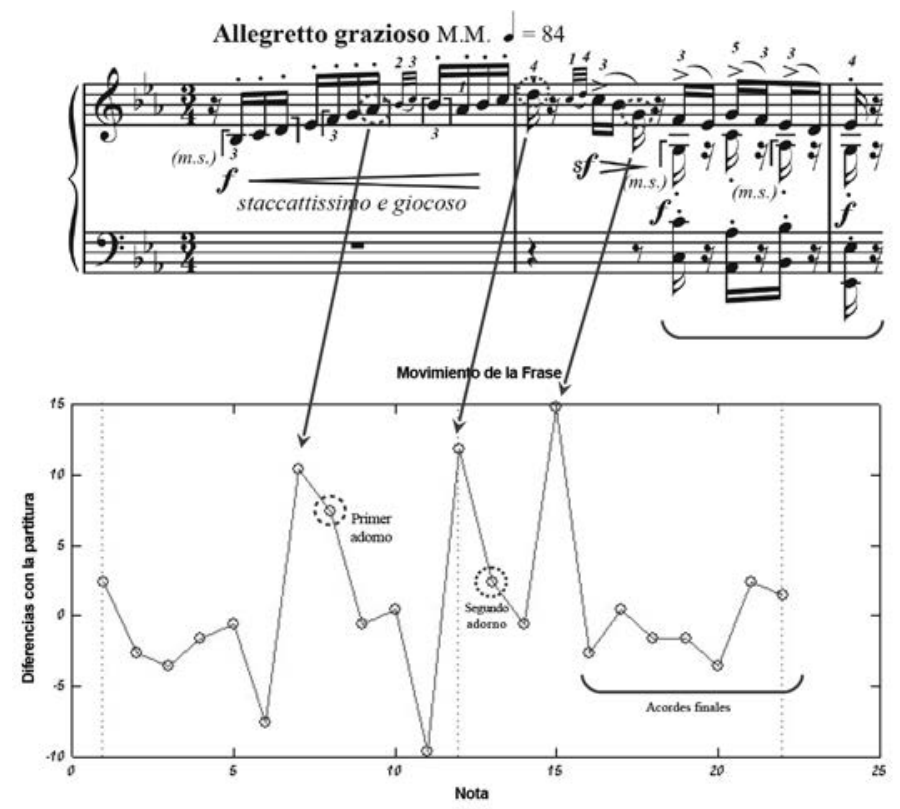

Fig. 30. 
Esta gráfica sugiere no precipitar los silencios, respirar antes de los adornos y, sumado a la imagen del espectrograma, una respiración en el segundo La bemol.
Para ver con mayor claridad algunos detalles de los acordes finales, he suprimido en la imagen algunos de los armónicos superiores del fragmento (fig. 31 .

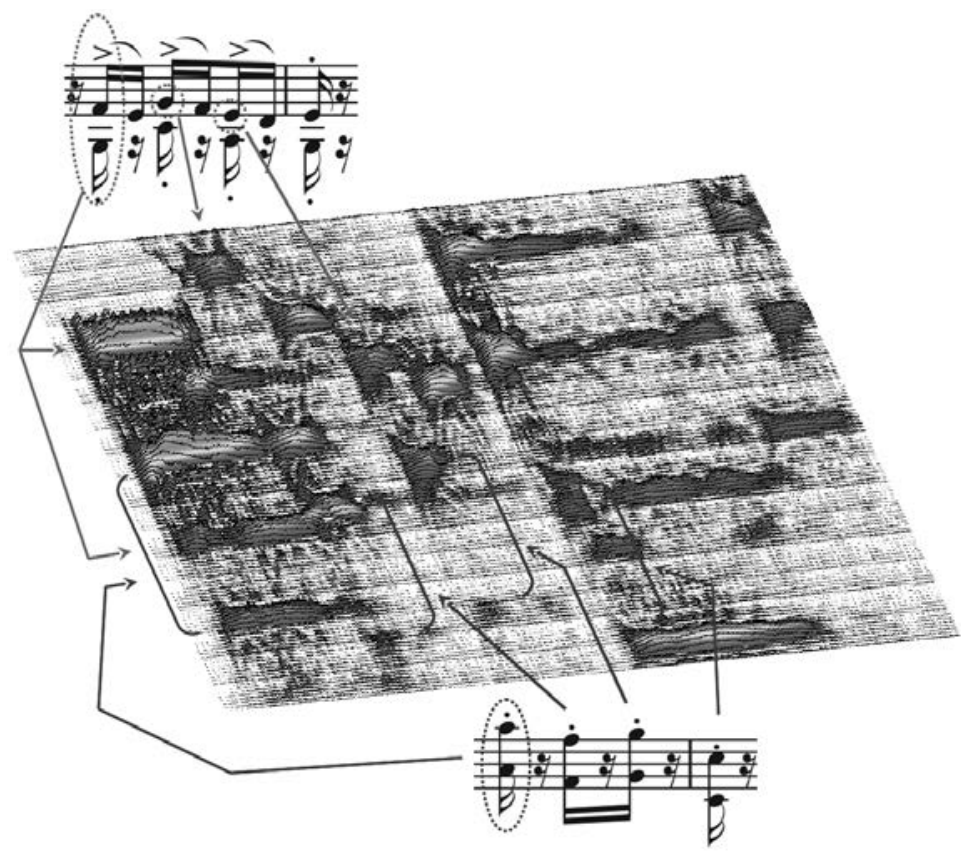

Fig. 31

El primer acorde está más marcado y ligado que los demás (la resonancia del Fa se superpone al Mib); la sensación de intensidad que se aprecia en la escucha se debe también en parte a la propia prolongación del sonido. La voz superior principal (Fa Mib Sol Fa Mib Re Mib) se produce ligeramente antes que las otras voces, muy probablemente, no por un problema de intencionalidad, sino del propio mecanismo del piano y sus asincronías, como he comentado anteriormente. Así, se observa, en la mano derecha, el cuidado de los segundos planos Sol, Do y La bemol que ha ejecutado más suavemente. Del mismo modo, en la mano izquierda, los bajos de las octavas están tocados con más suavidad, ya que sonido inferior se produce después que el superior, tocado con el pulgar.

La imagen también sugiere pedales únicamente en el primer acorde y en el de resolución, ya que el La bemol y el Si bemol de las octavas de la mano izquierda aparecen cortados, mientras que los otros aparece la resonancia más prolongada en el Sol, el Do y el Fa.

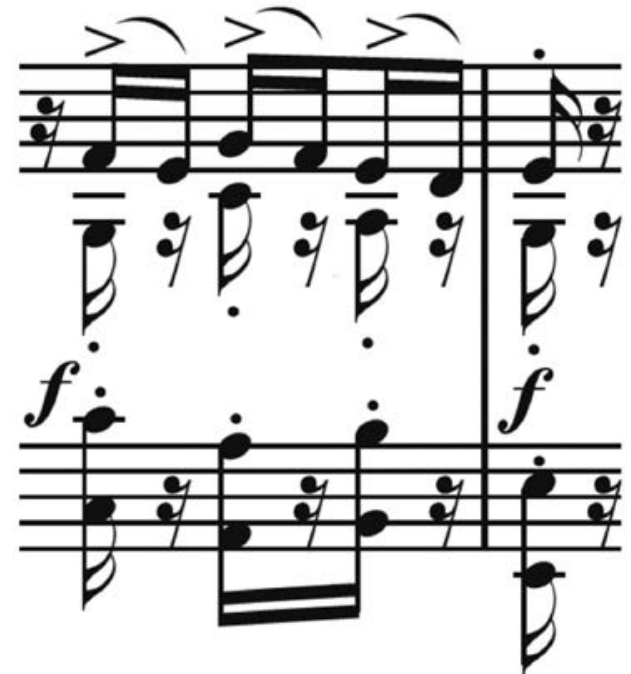

Fig. 32a 


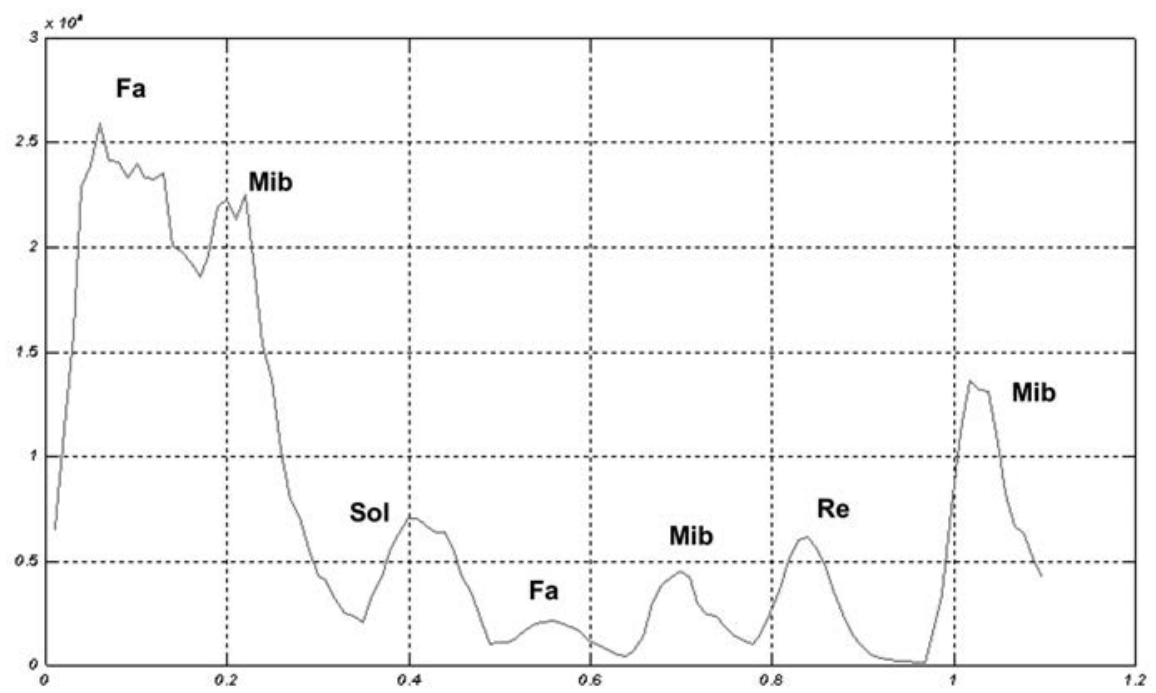

Fig. $32 b$

La envolvente de la señal (fig. 32b), muestra más claramente la dinámica del final. Los cuatro acordes avanzan en diminuendo, pero en el acorde final de la tónica, la pianista vuelve a subir la dinámica, atacando con más sonido. Desde mi punto de vista, en la percepción cuando se escucha, realiza más bien un mezzoforte que un forte; en la gráfica se observa que tiene menos energía, menos intensidad que el primer acorde de la serie y que está bien separado del resto.
Para finalizar, muestro en la partitura las indicaciones que se han observado y que corresponderían a la versión de Pilar Bayona (fig. 33). Éstas podrían servir también como sugerencia pedagógica para el estudio de esta introducción y una referencia para encontrar otras opciones de interpretación.

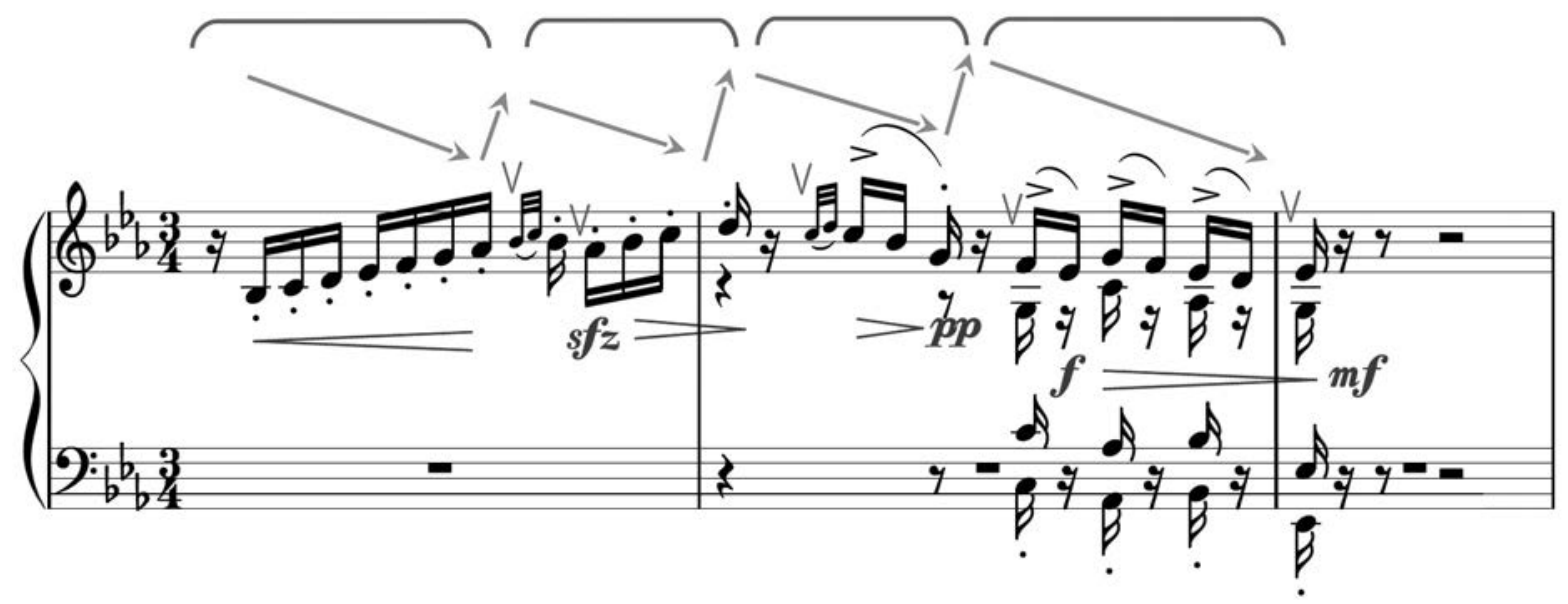

Fig. 33 
El fragmento está estructurado en cuatro partes delimitadas por las respiraciones y el ataque subsiguiente. Las indicaciones agógicas están indicadas con las flechas.

\section{CONCLUSIONES}

De los análisis de la pianista aquí expuestos, se pueden concluir algunas cuestiones.

En un análisis comparado con la partitura, las gráficas de fraseo muestran una organización de los movimientos de las frases asociadas a los elementos principales de estas. Se observa que la pianista integra las variaciones de ritmo en elementos pequeños específicos de las frases, organizando los puntos culminantes agógicos a lo largo de ella. Desde mi punto de vista, la gráfica del movimiento de los compases muestra en varias ocasiones, cómo la pianista realiza ritardandos de forma gradual, con elegancia. Todo esto vendría a demostrar una gran habilidad de la pianista para integrar el detalle en la forma más grande. Se ha observado característico de la pianista culminar las frases invirtiendo la dinámica, es decir, cuando el movimiento es ascendente, la dinámica es descendente.

Algunas observaciones sobre su manera de articular los sonidos demuestran que la duración del sonido y la manera de articular las voces tiene una clara influencia en la calidad y nitidez de los planos sonoros (Sonata de Soler).

Respecto a pasajes concretos, Pilar Bayona realiza una especial y personal caracterización rítmica, por ejemplo en los acordes de Lavapiés y en el comienzo de Eritaña. Como un rasgo típico de su fraseo se observa una tendencia al alargamiento de las partes débiles al final del compás.

Se han observado otros aspectos de interés sobre la realización mecánica del pianista, incluso pudiéndose inferir, en algún caso, una digitación o una pedalización.

Los espectrogramas muestran también una utilización discreta del pedal; en momentos concretos la pianista utiliza como recurso mantener pegados los dedos en las teclas, lo que le permite una mayor libertad en la utilización del pedal.

En otros casos, se han revelado modificaciones sustanciales de la intérprete sobre las indicadas en la partitura. Los espectrogramas han demostrado que la pianista realizaba, a veces, arreglos de pasajes de las obras, cambiando algunas notas de la partitura. Estos están realizados de tal manera, que pasan casi desapercibidos en la escucha, lo que demuestra un sutil conocimiento de los recursos del piano. A veces, añade notas para dar claridad a un pasaje concreto y, de esta manera, hacer más explícito lo que está implícito.

\section{BIBLIOGRAFÍA}

Albéniz, Isaac: Iberia. Cuaderno III, 3.Lavapiés. Madrid, Fundación Albéniz, 2014, [Ed. Luis Fernando Pérez].

Albéniz, Isaac: Iberia. Cuaderno IV, 3. Eritaña. Madrid, Fundación Albéniz, 2014, [Ed. Luis Fernando Pérez].

Bayona, Antonio; y Gómez, Julián: Pilar Bayona. Biografía de una pianista. Zaragoza, Prensas de la Universidad de Zaragoza [Col. De Arte], 2015.

Bayona de la Llana, Antonio; et alii: Pilar Bayona. La pasión de la música. Zaragoza, Cortes de Aragón, 2004.

Bayona, Pilar: Isaac Albéniz. Evocación, Eritaña. París, Lumen, 1955, [LDI 412].

Bayona, Pilar: Isaac Albéniz. Lavapiés, Málaga. París, Lumen, 1955, [LDI 414].

Broto Salamero, Julio: Diccionario Biográfico Musical Aragonés. Huesca, Gráficas Alós, 1986.

Derqui, Manuel: "La sonata española", en Sopeña Ibáñez, Federico: Pilar Bayona. Zaragoza, Institución "Fernando el Católico", 1982: 120-121.

Iglesias, Antonio: Óscar Esplá (su obra para piano). Madrid, Dirección General de Relaciones Culturales, 1962.

Iglesias, Antonio: Óscar Esplá, Joaquín Rodrigo, Rodolfo Halffter, Manuel de Falla, Isaac Albéniz, Cristóbal Halffter, Joaquín Turina, sus obras para piano y orquesta. Madrid, Alpuerto, 1994.

Menéndez Pidal, Ramón: Romancero hispánico (hispanoportugués, americano y sefardí). Teoría e historia. Madrid, Espasa-Calpe, 1953: 256.

Pérez-Lizano Forns, Manuel: Aragoneses rasgados. Zaragoza. Ibercaja. col. "Boira, 9". 1991.

Pérez-Lizano Forns, Manuel: Aragoneses rasgados. Zaragoza, Ibercaja, col. "Boira, 9", 1991, p.38.

Sopeña Ibáñez, Federico: Pilar Bayona. Zaragoza, Institución "Fernando el Católico", 1982.

Sopeña Ibáñez, Federico: Pilar Bayona. Zaragoza, Institución "Fernando el Católico", 1982: 10.

Soler Antonio: Sonata in D major, R.84. Montreal, Les Editions Outremontaines, 2006, [ed. Pierre Gouin ].

VV.AA.: Federico Sopeña y la España de su tiempo, 19391991. Libro homenaje. Madrid, Fundación Isaac Albéniz, 2000.

VV.AA.: Pilar Bayona 30 miradas. Juan Francisco Nevado (ed.). Zaragoza, Libros del Innombrable, 2009: 46.

Recibido: 23.01 .2016

Aceptado: 24.08.2017 